\title{
Relationship Between Intermittent Separation and Vortex Structure in a Three-Dimensional Shock/Boundary-Layer Interaction
}

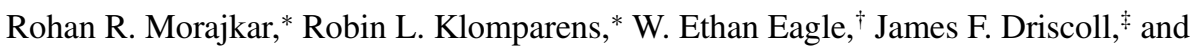 \\ Mirko Gamba \\ University of Michigan, Ann Arbor, Michigan 48109 \\ and \\ John A. Benek $\mathbb{I}$ \\ U.S. Air Force Research Laboratory, Wright-Patterson Air Force Base, Ohio 45433
}

DOI: $10.2514 / 1 . J 053905$

\begin{abstract}
The relationship between the three-dimensional vortex structures and flow-separation zones generated by a shock wave/boundary-layer interaction within a low-aspect-ratio duct was studied using stereoscopic particle imaging velocimetry measurements. In this configuration, the interaction of the incident shock with all walls was important in controlling the flowfield; the three interactions coupled to produce a strongly distorted flowfield. Conditional sampling was used to construct the local probability of reverse flow maps, and thus quantify the distribution of regions of intermittent separation on both bottom-walls and side-walls. The latter regions were found to be significantly larger and more likely to separate than the former. Thus, it was concluded that the sidewall and corner flow interactions dominate in this configuration. A triple decomposition of motion was used to construct a three-dimensional representation of the vortex features generated by the interaction. The results indicated that the flowfield was dominated by three vortex systems: 1) the vortex associated with the sidewall swept-shock interaction; 2) a complex, possibly branched, vortex pair induced on the bottom wall; and 3) a vortex pair induced by the flow at the corner, which coupled the two interactions. The role of the three vortex systems on the onset of flow separation was also explored and discussed.
\end{abstract}

\section{Nomenclature}

$A=$ flow-separation bubble cross-section area

$A_{T} \quad=$ one-quarter of the cross-sectional area of the duct

$\boldsymbol{H}=$ pure shear tensor of rotated velocity gradient tensor defined by the triple decomposition of motion method

$M \quad=$ Mach number

$P_{\infty} \quad=\quad$ undisturbed freestream static pressure

$\boldsymbol{R}=$ residual tensor of rotated velocity gradient tensor defined by the triple decomposition of motion method

$R_{\delta} \quad=\quad$ Reynolds number based on boundary-layer thickness; $\rho U_{\infty} \delta / \mu$

$R_{\theta} \quad=$ Reynolds number based on momentum thickness; $\rho U_{\infty} \theta / \mu$

$S \quad=\quad$ strain rate tensor

$\hat{\boldsymbol{S}}=$ strain rate tensor after pure shear is removed using the triple decomposition of motion method

$U_{\infty}=$ freestream streamwise velocity

$u=$ component of velocity along the $x$ direction

$v=$ component of velocity along the $y$ direction
Presented as Paper 2014-1138 at the 52nd AIAA Aerospace Sciences Meeting, National Harbor, MD, 13-17 January 2014; received 6 September 2014; revision received 31 July 2015; accepted for publication 15 November 2015; published online 19 April 2016. Copyright (C) 2015 by the authors. Published by the American Institute of Aeronautics and Astronautics, Inc., with permission. Copies of this paper may be made for personal and internal use, on condition that the copier pay the per-copy fee to the Copyright Clearance Center (CCC). All requests for copying and permission to reprint should be submitted to CCC at www.copyright.com; employ the ISSN 00011452 (print) or 1533-385X (online) to initiate your request.

*Graduate Student Research Assistant, Dept. of Aerospace Engineering. Student Member AIAA.

${ }^{\dagger}$ Currently Sandia National Laboratories, Livermore, CA 94550. Member AIAA.

\#Arthur B. Modine Professor, Dept. of Aerospace Engineering. Fellow AIAA.

${ }^{\S}$ Assistant Professor, Dept. of Aerospace Engineering. Member AIAA.

"Director, Computational Sciences Center, AFRL Aerospace Systems Directorate. Fellow AIAA.

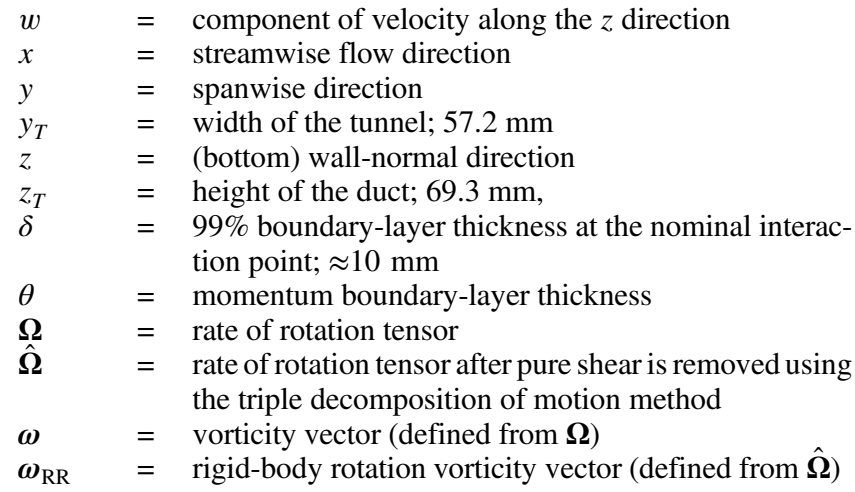

Subscripts

$w \quad=$ quantity evaluated at the wall

$x, y, z=$ derivative in respective direction

$\infty=$ quantity evaluated in the freestream

\section{Introduction}

$I^{\top}$ $\mathrm{N}$ THIS work, we investigate the properties of the highly intermittent three-dimensional (3-D) flow separation and the structure of the vortical features in a three-dimensional incident shock wave/ boundary-layer interaction (SBLI) generated in a low-aspect-ratio supersonic duct flow (width-to-height aspect ratio of 0.83). Because of the low aspect ratio, the SBLI is controlled by the shock interaction on both the sides and the floor of the duct. However, the interaction on the sidewall and at the corner dominates. Stereoscopic particle image velocimetry (PIV) measurements on several orthogonal planes distributed around the interaction region are used to extract conditional statistics $[1,2]$ on the location and shape of the intermittent separation. A triple decomposition of motion (TDM) [ $\underline{3}$ ] is used to identify regions of large rigid-body rotation vorticity (i.e., region of swirling flow), which are then used to construct a 3-D representation of the vortical features generated as a result of the shock interaction. 
Previous work [4] on this flow configuration used ensembleaveraged measurements and oilflow visualization combined with critical point theory to generate a morphological map of the flowfield, from which the possible structure of the vortical features was inferred. This work builds on and differentiates from that previous work to further assess and verify the structure of the flow and the separated regions using conditional statistics on the velocity field and TDM. The work is based on the database first constructed for this flow configuration [5] and later extended to include more measurement planes []].

The interaction considered in this study is relatively weak (6 deg deflection angle in a nominally Mach 2.75 duct flow) and results in incipient separation on both side-walls and bottom-walls [7]. A practical and scientific problem arises in this case if one wants to analyze the properties of the intermittent flow separation. Some of the velocity data from our experiment clearly show that separation is occurring; for example, the instantaneous three-component (3C) velocity fields indicate that reverse flow (i.e., negative streamwise velocity) occurs near the wall in local recirculation regions and that there is an abrupt increase in the mean velocity away from the wall. However, the ensemble-averaged $3 \mathrm{C}$ velocities display no locations with mean reverse flow. This is because the region of separated flow near the wall is oscillating in space and time. Ensemble-averaged data therefore fail to identify the locations and magnitudes of the separated regions. The formulation of maps of local probability of reverse flow and the computation of the separation bubble height $[1,2]$ are used to shed light on the unsteady properties of the separated flow regions in relation to the vortical features that exist in the flow.

There are many examples of flow separation for which the velocity field is intermittent and highly unsteady $[\underline{1}, \underline{2}, \underline{4}-\underline{6}]$. It is not surprising that Reynolds-averaged Navier-Stokes computations of these examples show poor agreement with experiments [8]. Large-eddy simulations offer a way to capture the unsteadiness and intermittency $[\underline{9}, \underline{10}]$. It is somewhat limited to compare values of ensemble-averaged velocity, since these values often do not indicate if the flow is separated or if they capture any flow property associated with the unsteadiness of the flow. Instead, parameters that represent a good choice for comparison include certain conditional statistics as well as probabilistic metrics quantifying separation and its properties.

The primary objective of this study is to establish the dominant flow structures such as the large-scale vortex tubes, flow-separation bubbles, and their behavior in a 3-D SBLI. A global structure of such vortices is then presented, and the physics responsible for their behavior and the effects they have on the flow downstream of the interaction are then discussed. Specifically, the present work provides new information on the intermittently separated 3-D SBLI where interaction on all walls of the duct controls the overall response of the flow. In particular, here, we provide 1) an assessment of the inability of ensemble-averaged and oilflow visualization data to identify the desired properties; 2 ) construction of conditional statistics to quantify the probability, spatial extent, and statistical properties of separated flow; 3 ) a comparative measure of the likelihood and relative size of separated flow on the bottom wall and side-walls; 4) demonstrations that the interaction on the sidewall dominates the overall interaction (in the sense that is mainly responsible for the modifications of the flowfield) and the crucial role of the corner flow on sidewall separation; 5) a 3-D plot of the measured rigid rotation vorticity pattern from which a representation of the vortical features that are consistent with the measured vorticity field is constructed and is argued to be one of the underlying mechanisms that controls the flow pattern.

\section{Previous Research}

Previous studies by Reda and Murphy [11], Bruce et al. [12], Eagle and Driscoll [4], and Benek et al. [13] have shown that, for a shock wave/boundary-layer interaction in a rectangular duct, the sidewall and bottom-wall separation regions will be coupled. Qualitative evidence was provided by surface oilflow and wall pressure data by Reda and Murphy [11] and Bruce et al. [12]. Recently, Eagle and Driscoll [4], Eagle [5], and Morajkar et al. [6] used stereo-PIV to record a comprehensive database that included the three components of velocity along 25 orthogonal measurement planes. However, there have been no attempts to determine the actual locations where separation begins and the relative sizes of the sidewall and bottom-wall regions.

Previously, Humble et al. [14] and Helmer et al. [15,16] established measurements of such a system by means of planar two-component PIV on selected planes. However, many previous efforts lacked complete 3-D flowfield measurements spanning the entire flowfield. In an attempt to fill this gap, the current database was established in a 3-D interaction occurring in a low-aspect-ratio duct flow in which shock interactions on all walls were important; thus, the overall interaction results from a coupling between the incident and the swept-shock SBLIs.

The physics of each unit problem in an unconfined configuration (compression ramp and incident oblique SBLIs) have been extensively studied throughout the years by researchers such as Korkegi [7], Adamson and Messiter [17], Zheltovodov [18], Dolling and Clemens [19], Kubota and Stollery [20], and Delery and Dussauge [21,22]; while Alvi and Settles [23], Lu [24], Panaras [25], and Knight et al. [26] have studied the swept-shock interaction, corner effects have been studied by Burton and Babinsky [26]. Numerous review papers on the same interactions have also been published $[27,28]$. However, relatively fewer studies have been conducted on 3$\overline{\mathrm{DS}} \overline{\mathrm{BL}}$ Is with coupled interactions in low-aspect-ratio ducts.

Bruce and Babinsky [8], Bermejo-Moreno et al. [9], Reda and Murphy [11], Benek et al. [13], Helmer et al. [17], Burton and Babinsky [26], Eagle et al. [29], and Morgan et. al. [30] established the importance of the sidewall in three-dimensional low-aspect-ratio duct flows. Previous studies describing the importance of corners in SBLIs have been reported for various configurations, such as those by Bruce et al. [12], Burton and Babinsky [26], Eagle et al. [29], Batcho and Sullivan [31], and Cresci et al. [32], whereas recently, the oilflow studies conducted by Doerffer and Dallmann [33] have given an insight into the flow structure resulting from a shock/corner flow interaction. Reda and Murphy [11] were among the first to note the regions of separations observed near the corners in such flows. Bruce et al. $[12,34]$ and Benek et al. [13] established the importance of the aspect ratio of the facility in determining the possibility of flow separation in a SBLI. Handa et al. [35] conducted two-dimensional (2-D) measurements and computations of a 3-D normal SBLI problem; unfortunately, 2-D measurements are not sufficient to generate a complete picture of the dominant dynamics. Helmer et al. [16] conducted 2-D PIV measurements in similarly oriented data planes, which again suffered from the same limitation. Recently, Humble et al. [36] presented tomographic PIV measurements that made it possible to view the instantaneous flow structures existing in the interaction region; however, their measurement domain did not span the entire flowfield to extract the structures dominating the flow on larger scales. Recently, the computational work of Morgan et al. [37], Bermejo-Moreno et al. [9], and Wang et al. [38] demonstrated the crucial importance of including sidewall and corner regions in order to correctly capture the properties of a normal shock train flow.

\section{Experimental Facilities}

\section{A. Supersonic Wind-Tunnel Facility}

The work presented in this paper was conducted in our glass windtunnel facility $[39,40]$. A schematic diagram of it is shown in Fig. 1. It is a vacuum-driven supersonic wind tunnel with a cross section of $57.2 \times 69.3 \mathrm{~mm}(2.25 \times 2.75 \mathrm{in}$. $)$, which results in a width-to-height aspect ratio of 0.83 . The nominal operational Mach number is 2.75 , with the nominal stagnation pressure and temperature near room conditions. The measured effective conditions of operation along with corresponding uncertainties are summarized in Table 1 . The uncertainties given in the table include both run-to-run variability and measurement accuracy. Error propagation rules have been used to estimate the uncertainties of derived quantities. Figure 1 also shows the right-handed coordinate system used throughout this work. The origin of the streamwise direction (i.e., $x=0$ ) is defined to be at the leading edge of the shock generator (to be discussed subsequently); the $y$ axis is perpendicular to the sidewall, whereas the $z$ axis is perpendicular to the bottom wall. The origin of the $y-z$ plane is centered at the right lower corner of the duct as one looks downstream. 


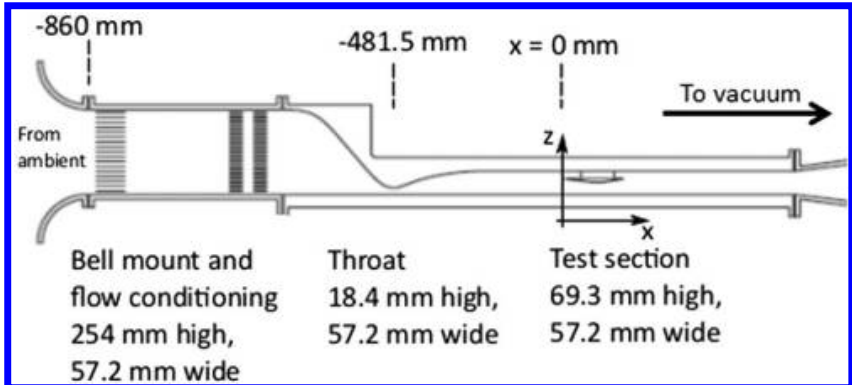

Fig. 1 Schematic diagram of the supersonic wind tunnel with relevant dimensions.

The supersonic wind tunnel has a one-sided converging-diverging nozzle that terminates in a constant-area 0.85 -m-long test section. Details of the design of this facility were given by Lapsa [39]. This configuration was originally selected to minimize pressure gradient history effects on the boundary layer developing on the bottom wall (floor) of the wind tunnel. The resulting boundary layer was found to be similar to an equilibrium flat-plate boundary layer, as described by Lapsa and Dahm [40]. The unit Reynolds number of the experiment was $8.9 \times 10^{6} / \mathrm{m}$ with an incoming boundary-layer thickness $\delta$ of $10 \mathrm{~mm}$, measured by Lapsa and Dahm [40] at $x=76 \mathrm{~mm}$ in an empty tunnel; this location was upstream of the nominal location of the bottom-wall interaction, which was found at $x=96 \mathrm{~mm}$, as will be explained in the following. In spite of being upstream of the interaction point, we referred to this quantity to as the "boundarylayer thickness at the nominal interaction point," and we used it as a relevant length scale to normalize some of the quantities presented here. Optical access to the test section was provided from both sides of the wind tunnel by glass windows that ran along the whole length of the wind tunnel, including the nozzle-throat region. Optical access through the floor was also provided by a flush-mounted acrylic window located at the nominal location of the incident shock.

An oblique shock wave is generated by a 6 deg full-span wedge mounted on the top wall, as indicated in Fig. 1. The resulting oblique shock wave spans the full width of the wind tunnel and impinges on its floor. As a result, the oblique shock interacts with both the boundary layer on the bottom wall and the side-walls of the wind tunnel as a classical incident and swept SBLI, respectively. Although this complex interaction exists for most, if not all, configurations studied in the literature, the low aspect ratio of this wind tunnel emphasizes this complex interaction. The wedge geometry is shown in Fig. 2, where relevant dimensions (in millimeters) are reported. The wedge is suspended by a strut (see Fig. 2), such that the leading

Table 1 Summary of experimental conditions

\begin{tabular}{|c|c|}
\hline$\underline{\text { Parameter }}$ & Value \\
\hline Mach number $M_{\infty}{ }^{a}$ & $2.70 \pm 0.01$ \\
\hline Mach number $M_{\infty}{ }^{\mathrm{b}}$ & $2.72 \pm 0.03$ \\
\hline Freestream velocity $U_{\infty}{ }^{\mathrm{c}}$ & $593 \pm 2 \mathrm{~m} / \mathrm{s}$ \\
\hline Stagnation pressure & $98.5 \pm 0.2 \mathrm{kPa}$ \\
\hline Stagnation temperature & $294 \pm 2 \mathrm{~K}$ \\
\hline Wall static pressure $(x=-85 \mathrm{~mm})^{\mathrm{d}}$ & $4.15 \pm 0.05 \mathrm{kPa}$ \\
\hline Static temperature $T_{\infty}{ }^{\mathrm{c}}$ & $119 \pm 1 \mathrm{~K}$ \\
\hline Wedge leading edge & $x=0 \mathrm{~mm}$ \\
\hline Mean center of bottom-wall separation & $x=96 \mathrm{~mm}$ \\
\hline Test section height ${ }^{\mathrm{d}}$ & $69.3 \pm 0.2 \mathrm{~mm}$ \\
\hline Test section width ${ }^{d}$ & $57.2 \pm 0.1 \mathrm{~mm}$ \\
\hline Throat height $\mathrm{d}^{\mathrm{d}}$ & $18.4 \pm 0.1 \mathrm{~mm}$ \\
\hline Throat-to-wedge leading-edge distance ${ }^{d}$ & $481.5 \pm 1 \mathrm{~mm}$ \\
\hline
\end{tabular}

${ }^{\mathrm{a} C}$ Computed from a direct measure of stagnation and static pressure assuming isentropic expansion $(\gamma=1.4)$

${ }^{b}$ Computed from a direct measure of the ratio of stagnation pressure and test section pitot pressure $(\gamma=1.4)$

Uncertainty estimated from error propagation rule.

${ }^{\mathrm{d}}$ Uncertainty includes both measurement accuracy and day-to-day variability.

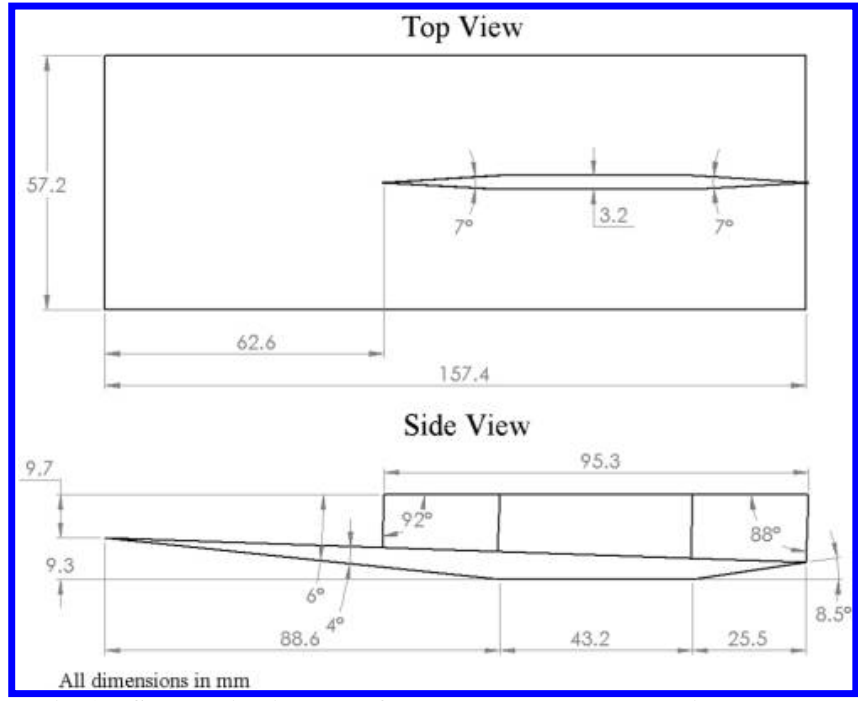

Fig. 2 Schematic diagram of the wedge geometry used in the study.

edge of the wedge is $9.7 \mathrm{~mm}$ below the top wall of the wind tunnel and outside of the top-wall boundary layer. The leading edge of the shock generated is located $482 \mathrm{~mm}$ downstream of the nozzle's throat, and it is taken to be at $x=0$ in our coordinate system. The oblique shock wave thus formed is anchored very steadily to the leading edge of the wedge and is incident on the bottom wall on the centerplane of symmetry of the tunnel at a streamwise distance of $96 \mathrm{~mm}$ from the leading edge (i.e., at $x=96 \mathrm{~mm}$ ). However, owing to the shock's curved nature, the point of impingement moves upstream as one moves away from the centerplane. We will discuss shock curvature in Sec. IV.B. The upper surface of the wedge diverges by 2 deg in order to prevent choking in the channel formed between the wedge and the top wall by providing expansion.

The supersonic wind-tunnel facility was equipped with a standard folded $z$-type schlieren system. A continuous high-intensity mercury lamp was used for illumination. A pair of spherical mirrors with a focal length of $1.5 \mathrm{~m}$ was used to collimate and focus the light. A high-speed complementary metal oxide silicon (CMOS) camera (Vision Research Phantom V711) operated at 9000 frames per second with $5 \mu \mathrm{s}$ of exposure was used to acquire high-speed movies of the flowfield during the run of the wind tunnel.

\section{B. Stereoscopic Particle Image Velocimetry Arrangement}

Stereoscopic PIV measurements were made using the setup shown in Fig. 3. Two interline transfer charge-coupled device cameras (SensiCam PCO) recording at $3.33 \mathrm{~Hz}$ with a resolution of $1280 \times$ 1024 pixels were used in a forward-scattering stereoscopic mode. The cameras were oriented at 33 deg relative to the measurement plane. The cameras were equipped with a Sigma 70-300f/4 - 5.6 apochromatic (APO) macro lens. The double-pulse illumination of the flow was provided by a pair of low-repetition-rate frequencydoubled Nd:YAG lasers producing an output of a $532 \mathrm{~nm}$ beam with a total energy of $200 \mathrm{~mJ} /$ pulse. The lasers were triggered at $10 \mathrm{~Hz}$ with a time delay of $600 \mathrm{~ns}$ between the two pulses and with a pulse duration of about $10 \mathrm{~ns}$. The effective time delay between pulses was measured with a Thorlabs DT10A/M photodiode ( $1 \mathrm{~ns}$ response time) and a digital oscilloscope (LeCroy Waverunner 6030, $350 \mathrm{MHz}$ ), and it was adjusted to the desired valued by adjusting the nominal time delay between the trigger signals to the lasers. In Fig. 3 L1 refers to a cylindrical lens focusing in the horizontal plane, L2 is a cylindrical lens expanding the beam in the vertical plane and L3 is a focusing cylindrical lens collimating the beam in the vertical plane.

To optimize the particle dropout and volumetric effects, the laser sheet thickness was set using expanding-collimating optics to approximately four times the expected maximum out-of-plane displacement of a particle within the measurement domain, as suggested by Adrian and Westerweel [41]. The beam width was measured using 


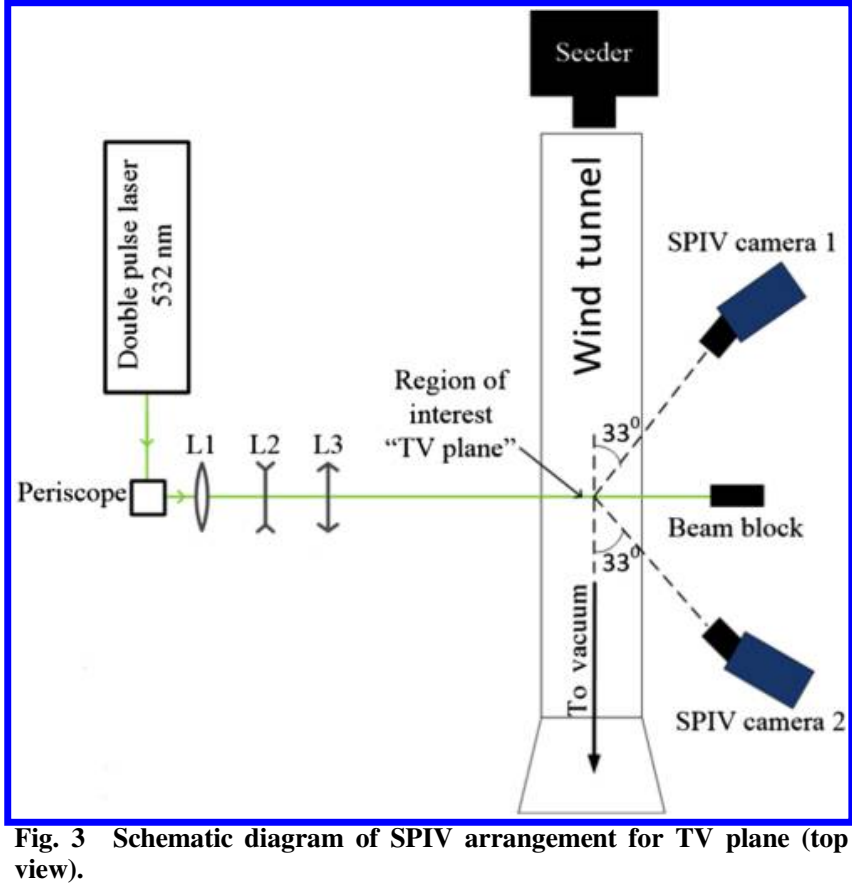

the scanning knife-edge method to be $1.25 \pm 0.25 \mathrm{~mm}$, which is approximately four times the particle displacement in the freestream (approximately $300 \mu \mathrm{m}$ ) in $600 \mathrm{~ns}$. Particle seeding of the flow was generated by a TDA-4B portable Laskin nozzle aerosol. The generator consisted of an array of six Laskin nozzles that created polydispersed submicrometer particles using poly-alpha olefin oil with a density of $819 \mathrm{~kg} / \mathrm{m} \mathrm{[3]}$.

The LaVision DaVis 7.2 and DaVis 8 software versions were used for the acquisition of the measurement and the processing of the data. The three-component velocity fields were reduced from the particle images using the DaVis software using a multipass scheme with window deformation and offset. Two passes were first conducted with a $64 \times 64$ pixel window size with $50 \%$ overlap, followed by two passes at a reduced $32 \times 32$ pixel window size with Gaussian weighting and $50 \%$ overlap. The final $32 \times 32$ pixel window size corresponded to a projected physical size ranging from about $0.2 \times 0.2 \mathrm{~mm}$ to $0.7 \times 0.7 \mathrm{~mm}$, depending upon the measurement plane orientation and location (the resolution on each plane is summarized in Table 2). Vectors were validated using the DaVis software using the median test. Valid vectors were found more than $95 \%$ of the time. Missing or rejected vectors were interpolated using the method by Garcia [42].

Three-component velocity measurements were made on several planes parallel to the three principal orthogonal planes. Here, we refer to them as streamwise-horizontal ( $\mathrm{SH})$ planes, streamwise-vertical (SV) planes, and transverse-vertical (TV) planes. In particular, SH planes are planes parallel to the $x-y$ plane; SV planes are parallel to $x-z$ planes; and TV planes are parallel to $y-z$ planes. Figure 4 shows the locations of the data planes with respect to the global configuration and theoretical (inviscid) location of the incident and reflected shocks, as well as the expansion fan. A summary of the location, dimension, and spatial resolution of different stereoscopic particle image velocimetry (SPIV) data planes is given in Table 2. Planes TV1 and TV2 are located well upstream of the location of the shock generator, whereas plane TV3 is located at the leading edge of the shock generator (i.e., $x=0$ ). These three initial planes are used to define the initial flow entering the interaction region that evolves further downstream. The remainder of the TV planes and all other planes are located around the nominal interaction point, which is here defined to be at $x=96 \mathrm{~mm}$.

The velocity fields obtained from the DaVis were then analyzed using the PIVMAT toolbox [43] to obtain various scalar and vector fields, such as mean velocity components, components of the strain rate tensor, Reynold's stress gradients, and root-mean-square fluctuation velocity components. The data were low-pass filtered and missing vectors interpolated using the smoothing-interpolating method of Garcia [42], which is a penalized least-squares method based on the method by Whittaker [44] and on discrete cosine transform.

\section{Pressure Measurements}

Static pressure measurements were obtained at bottom-wall and sidewall locations instrumented with pressure taps. Pressure tap sizing was based on the results of Mckeon and Smits [45], Chue [46], and Shaw [47] in order to minimize measurement errors and the effects of the presence of wall pressure tap on the fluid flow. Recessmounted pressure taps were used where the tap diameter and length were 0.8 and $7.6 \mathrm{~mm}$, respectively (depth-to-diameter ratio of approximately nine); stainless-steel tubing (inner diameter of $0.8 \mathrm{~mm}$, and outer diameter of $1.6 \mathrm{~mm}$ ) was press-fitted into the walls to connect the pressure tap to the pressure transducer. Different tap spacings were used: $25 \mathrm{~mm}$ upstream of the interaction, $6.35 \mathrm{~mm}$ around the interaction region, and $12.7 \mathrm{~mm}$ downstream of the interaction. Tygon B-44-4X flexible tubing (inner diameter of $1.6 \mathrm{~mm}$, and outer diameter of $3.2 \mathrm{~mm}$ ) was used to connect the pressure taps to a differential pressure sensor array scanner (Scanivalve model DSA 3217). The accuracy of the pressure sensor array was $0.009 \mathrm{kPa}$.

A reference wall pressure tap was located on the top wall at $x=-85 \mathrm{~mm}$. Measurements at this location were used to reference and monitor all runs (see also Table 1), as well as to reference all other differential measurements. Absolute pressure measurements were performed at this location with two different transducers: a pressure gauge (Omega model DPG2001B-30A, with an accuracy of $0.5 \mathrm{kPa}$ ), or a vacuum capacitance manometer (MKS Baratron 627D, with an accuracy of $0.12 \%$ of the reading). All pressure measurements reported here are time-averaged measurements over a minimum averaging time of $30 \mathrm{~s}$.

Because the test section has a constant area, the static pressure increases along the (empty) tunnel as a result of boundary-layer growth. The increase in pressure is quantified by the pressure gradient parameter defined as $\beta=\left(\delta / \rho U_{\infty}^{2}\right)(\partial P / \partial x)$ and was calculated from the sidewall pressure measurements conducted in the empty tunnel at half the tunnel height. The value of this parameter was found to be $5 \times 10^{-4}$ for this experiment.

Table 2 Summary of data planes

\begin{tabular}{lcccc}
\hline \hline Plane & $x, \mathrm{~mm}$ & $Y, \mathrm{~mm}$ & $z, \mathrm{~mm}$ & Resolution, mm \\
\hline \multicolumn{5}{c}{ Transverse-vertical planes $\Delta y \times \Delta z$} \\
TV1 & -144 & $1.3-45$ & $1.3-68$ & $0.648 \times 0.648$ \\
TV2 & -72 & $1.8-44$ & $1.6-68$ & $0.637 \times 0.637$ \\
TV3 & 0 & $1.2-4.5$ & $1.2-38$ & $0.602 \times 0.602$ \\
TV4 & 71 & $1.5-35$ & $1.1-27$ & $0.213 \times 0.213$ \\
TV5 & 76 & $1.5-35$ & $1.1-27$ & $0.213 \times 0.213$ \\
TV6 & 81 & $1.5-35$ & $1.1-27$ & $0.213 \times 0.213$ \\
TV7 & 86 & $1.5-35$ & $1.1-27$ & $0.213 \times 0.213$ \\
TV8 & 91 & $1.5-35$ & $1.1-27$ & $0.213 \times 0.213$ \\
TV9 & 96 & $1.5-35$ & $1.1-27$ & $0.213 \times 0.213$ \\
TV10 & 101 & $1.1-45$ & $1.2-41$ & $0.338 \times 0.338$ \\
TV11 & 107 & $1.6-45$ & $1.4-35$ & $0.576 \times 0.576$ \\
TV12 & 115 & $1.6-44$ & $1.4-41$ & $0.677 \times 0.677$ \\
TV13 & 130 & $1.8-44$ & $1.5-41$ & $0.677 \times 0.677$ \\
TV14 & 137 & $1.5-45$ & $1.2-41$ & $0.683 \times 0.683$ \\
TV15 & 145 & $1.5-45$ & $1.2-41$ & $0.683 \times 0.683$ \\
TV16 & 150 & $1.5-45$ & $1.2-41$ & $0.683 \times 0.683$ \\
TV17 & 155 & $1.6-45$ & $1.4-41$ & $0.683 \times 0.683$ \\
\multicolumn{5}{c}{ Streamwise-vertical planes $\Delta x \times \Delta y$} \\
SV1 & $72-122$ & 28 & $1.6-28$ & $0.603 \times 0.603$ \\
SV2 & $51-96$ & 21 & $1.2-30$ & $0.298 \times 0.298$ \\
SV3 & $61-113$ & 17 & $1.2-30$ & $0.631 \times 0.631$ \\
\multicolumn{5}{c}{ Streamwise-horizontal planes $\Delta x \times \Delta y$} \\
SH1 & $91-141$ & $14-45$ & 9.5 & $0.623 \times 0.623$ \\
SH2 & $77-125$ & $11-42$ & 19 & $0.631 \times 0.631$ \\
SH3 & $59-107$ & $12-44$ & 29 & $0.652 \times 0.652$ \\
\hline \hline
\end{tabular}




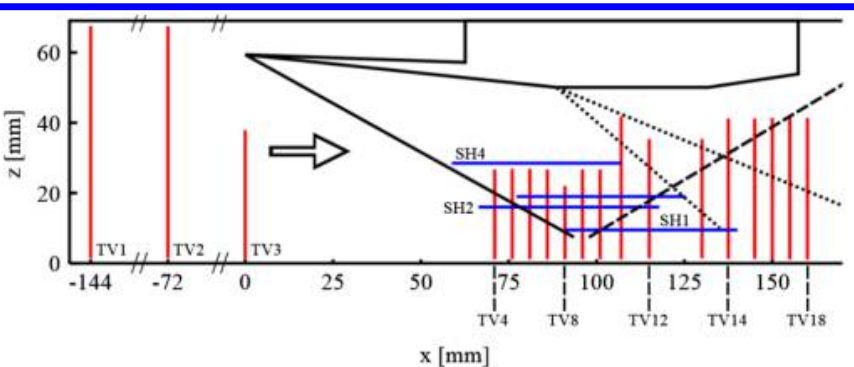

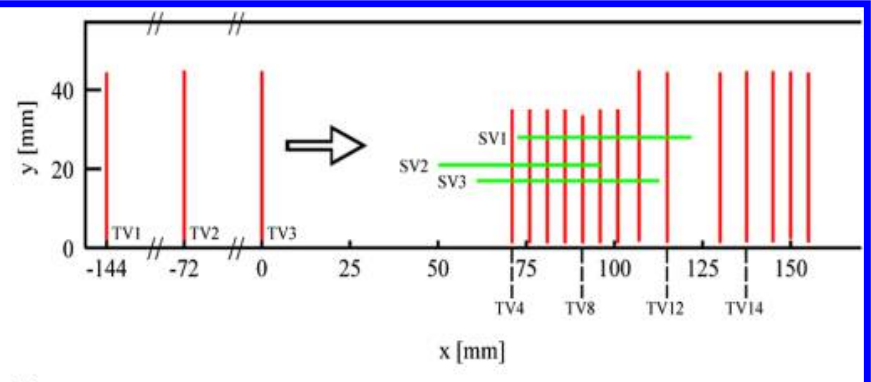

b)

Fig. 4 Location of SPIV planes: a) side and b) top view of the test section.

\section{Analysis and Results}

\section{A. Incoming Mean Flow}

To set the stage for the observations that will be discussed, and because it is important to understand the incoming baseline flow, we will first briefly describe the properties of the flow generated by the wind tunnel coming into the interaction region.

Figure 5 shows a time-averaged schlieren image of the flow around the interaction region where the main flow features are labeled and introduced in the discussion that follows. $C_{1}$ and $C_{2}$ indicate the incident and reflected shock waves, respectively; $E$ indicates the expansion wave from the shoulder of the shock generator; $W$ is the expansion wave associated with the interaction; and $U$ is the upstream shock that results from the disturbed (thickened and/or separated) boundary layer, which continues as the reflected shock $C_{2}$. I is the nominal interaction point, which is defined as the intersection point of $C_{1}$ and $C_{2}$. D is a weak disturbance wave generated by a slight mismatch between the floor and the bottom access window; this wave is found to be inconsequential to the flow properties. The expansion wave $E$ is sufficiently downstream of the interaction so that it does not disturb the flow at the interaction point I.

The wedge that generates the incident oblique shock is positioned such that the incident shock reaches the nominal interaction point nearly $582 \mathrm{~mm}$ downstream of the throat; this location corresponds to $x=96 \mathrm{~mm}$ in our coordinate system (plane TV9). Lapsa [39] showed that this location was sufficiently downstream of the throat for the incoming turbulent boundary layer to reach an equilibrium state with properties similar to those of a zero-pressure-gradient flat-plate turbulent boundary layer. In particular, Lapsa and Dahm $[39,40]$ performed high-resolution stereoscopic PIV measurements in the empty tunnel at two downstream locations to characterize the boundary-layer properties. Just upstream of the location of the nominal interaction point, and specifically at $x=76 \mathrm{~mm}$ (which corresponded to the location of plane TV7), they estimated a (99\%) boundary-layer thickness $\delta$ of $10 \mathrm{~mm}$ with a friction velocity of $u_{\tau}=30.9 \mathrm{~m} / \mathrm{s}$. This corresponds to local Reynolds numbers of $R e_{\delta}=89,000$ and $R e_{\theta}=9600$, where the momentum thickness $\theta$ here is based on the incompressible form.

Their assessment, however, was only limited to the state of the boundary layer near the symmetry plane of the test section. Because of the importance of the incoming flow to the properties of the

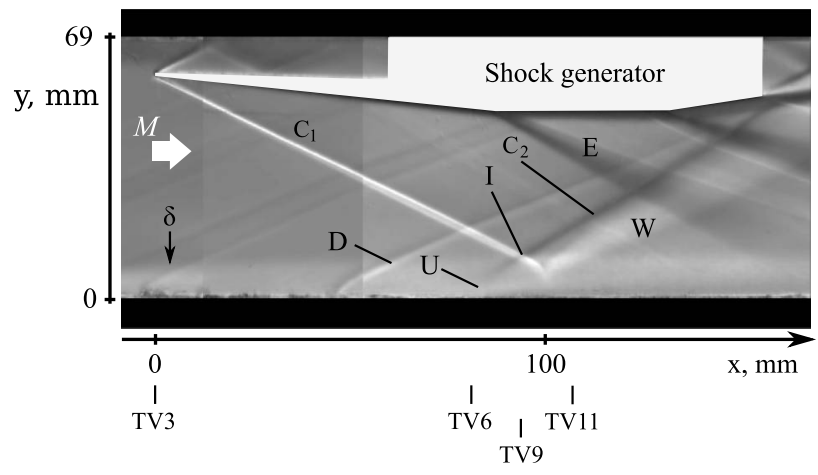

Fig. 5 Average schlieren image of the SBLI (side view). interaction, the properties of the incoming flow, and particularly near the corner and sidewall, will be investigated first. Planes TV1, TV2, and TV3 are well upstream of the interaction region, and therefore can serve to quantify the state of the incoming flow, as well as serve as inflow boundary conditions for possible computational fluid dynamics computations. Planes TV1 and TV2 span the full width and height of the wind tunnel, whereas plane TV3 only spans the lower right quadrant (as one looks downstream) and is located at $x=0$ (i.e., at the leading edge of the shock generator). As the TV3 plane is the closest of these three undisturbed planes to the interaction region, it will be discussed in more detail here. Although we, here, discuss only the mean fields, the statistics of the turbulence quantities are also available but not discussed here, and they can be made available to the interested reader.

The mean velocity field on the TV3 plane is shown in Fig. $\underline{6}$, where the mean in-plane velocity components are shown as vectors and the out-of-plane component (streamwise velocity) is shown as a color contour. The solid black line indicates the local (99\%) boundarylayer thickness. All velocity components shown here are normalized by the freestream undisturbed value $U_{\infty}$. The flow outside of the boundary layer is uniform. The boundary layers on the bottom-walls and side-walls develop differently. A similar trend is observed for the TV1 and TV2 planes, although the nonuniform corner region grows in size from TV 1 to TV3. Wall-normal and spanwise streamwise (mean) velocity profiles taken at different distances from the sidewalls and bottom-walls are shown in Figs. 7a and 7b, respectively. On the bottom wall, the boundary layer remains similar to the centerline profile up to $y=11.5 \mathrm{~mm}$; its $(99 \%)$ thickness varies from about $7.7 \mathrm{~mm}$ on the centerline to $8.5 \mathrm{~mm}$ at $y=11.5 \mathrm{~mm}$. Conversely, the boundary layer on the sidewall has a similar profile in the upper portion of the plane $(z>34.6 \mathrm{~mm})$, but it quickly thickens as the corner $(y=0, z=0)$ is approached. In particular, the sidewall boundary layer is $6.4 \mathrm{~mm}$ thick at $z=34.6 \mathrm{~mm}$ and $11.1 \mathrm{~mm}$ at $z=10.5 \mathrm{~mm}$. Thus, the sidewall boundary layer is thinner $(\sim 17 \%)$

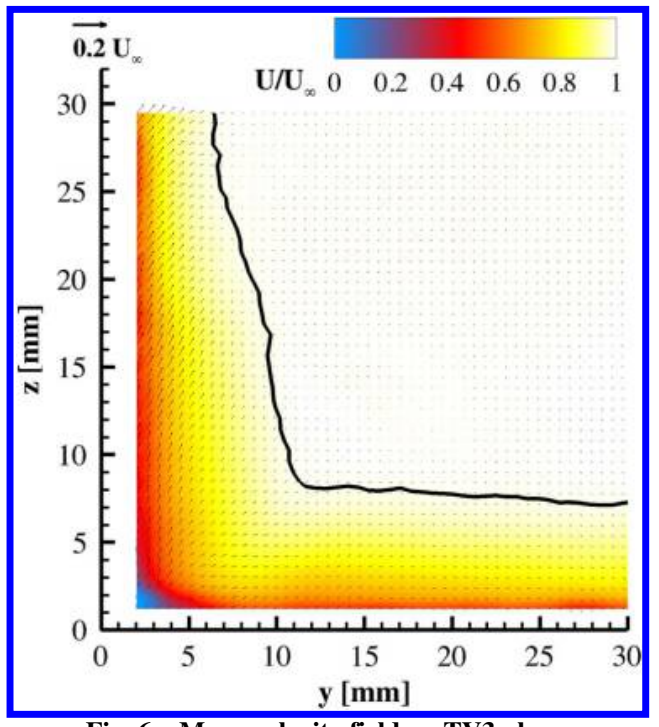

Fig. 6 Mean velocity field on TV3 plane. 


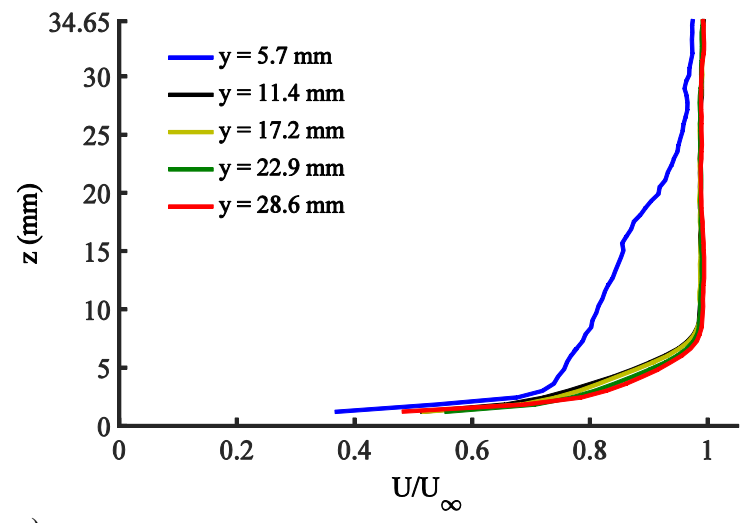

a)

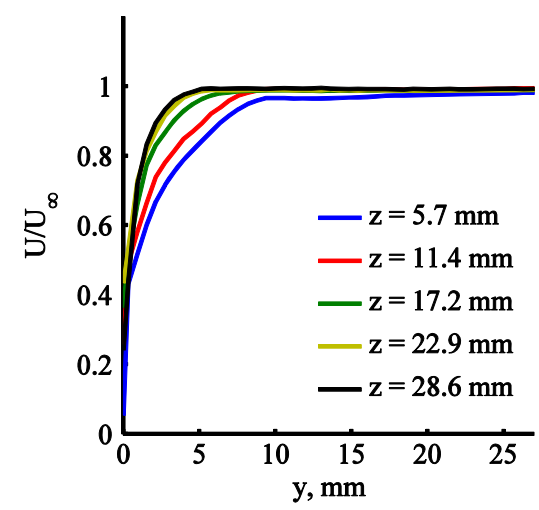

b)

Fig. 7 Mean streamwise velocity profiles on plane TV3 at different a) $y$ and b) $z$-locations.

than that on the bottom wall far from the corner but thicker as the corner is approached. The difference in boundary-layer thickness between the side-walls and bottom-walls is possibly due the onesided nature of our nozzle, which induces different pressure time histories and gradients experienced by the boundary layer on the sidewalls and bottom-walls, and thus result in different boundary-layer profiles on the different walls of the tunnel [48].

\section{B. 3-D Mean Flowfield Around the Interaction Region and Shock Structure}

In this section, we will discuss the global flow structure of the interaction from ensemble-averaged velocity fields. Figure $\underline{8}$ shows the mean velocity field on two SV planes where the streamwise velocity is indicated in the color contour with superimposed in-plane streamlines. The sonic line, which is here defined as the isocontour line of streamwise velocity equal to the speed of sound evaluated at the stagnation temperature, is also shown as a dashed line for reference as a convenient way to approximately indicate the extent over which the boundary layer is affected by the interaction: no particular physical meaning is necessarily attached to this isocontourline. Figure 8a shows the SV1 plane, which is on the centerplane of the duct and is centered on the interaction region (I in Fig. 5). On this plane, the overall flowfield resembles the classical two-dimensional interaction described by Babinsky and Harvey [49] and Délery and Dussauge [21], and it is generally consistent with the schlieren image of the flow in Fig. 5. The labels in Fig. 8a follow the flow features described in Fig. 5. Note that the ensemble-averaged field presented here is compiled from all realizations and does not show mean separation on the centerplane (at least within the measurement region).

In the SV3 plane (Fig. $\underline{\text { b) }}$ ), which is located at about $y=17 \mathrm{~mm}$ from the sidewall, the overall flow structure begins to change as a consequence of the sidewall, as observed by Helmer et al. [16]. In particular, the effect of the reflected shock begins to smear out.
Furthermore, the projected point of incidence of the incident shock on the bottom wall moves upstream relative to SV1 (from about $x=$ $120 \mathrm{~mm}$ to $x=110 \mathrm{~mm}$, respectively), although the shock angle remains unchanged. It has to be pointed out that both the incident and reflected shock angles are somewhat larger than the inviscid solution, whereas the flow deflection past the incident shock is estimated from the velocity measurements to be about $5.2 \mathrm{deg}$ on centerline and reduces to $3.8 \mathrm{deg}$ as the wall is approached ( $y=17 \mathrm{~mm}$ plane). Furthermore, the two-dimensional streamlines on planes approaching the sidewall becomes curved past the incident shock, unlike inviscid theory would predict. This deviation, which is contrary to the observation by Helmer et al. [16], is most likely due to the lateral flow effects caused by the swept-shock/corner flow interactions.

The free interaction theory [50] predicts that, for our flow configuration, the pressure increase across the interaction region for incipient flow separation is about 2.2: a pressure ratio which is also equal to the value of an inviscid 2-D interaction. A similar conclusion is also valid for the sidewall interaction that is described by Korkegi [51]. Figure 9 shows the pressure distribution measured along the wind tunnel at different locations on the bottom-walls and side-walls. Consider first the pressure profile measured on the bottom wall. It was measured along the centerline (corresponding to SV1; Fig. 8a). The wall pressure is seen to rise smoothly across the interaction region, peaks at a value of about 2.3 near $x=105 \mathrm{~mm}$, drops and remains constant at about 2.2 up to $x=140 \mathrm{~mm}$, and then decreases. The decrease afterward is attributed to the presence of the expansion wave originating from the end of the compression surface on the shock generator (see $E$ in Fig. 5). Therefore, although significant thickening of the boundary layer near the centerline is observed, mean flow separation does not occur. In this respect, the interaction can be classified as weak on the centerline; overall, it has features similar to a purely two-dimensional interaction. We will see, however, that, overall, the interaction is highly three-dimensional and the properties of the interaction on the SV1 plane are the result of SV1

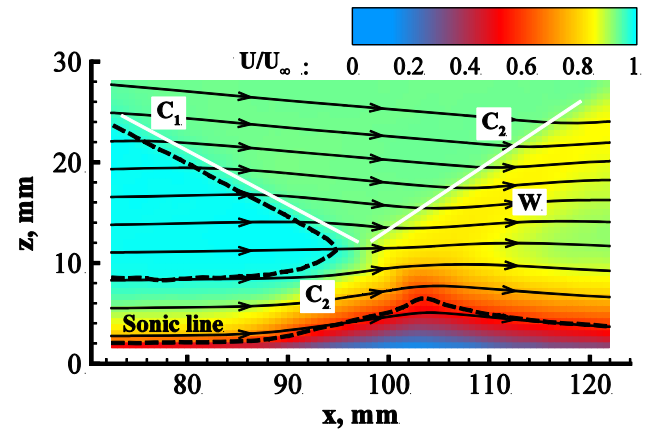

a)

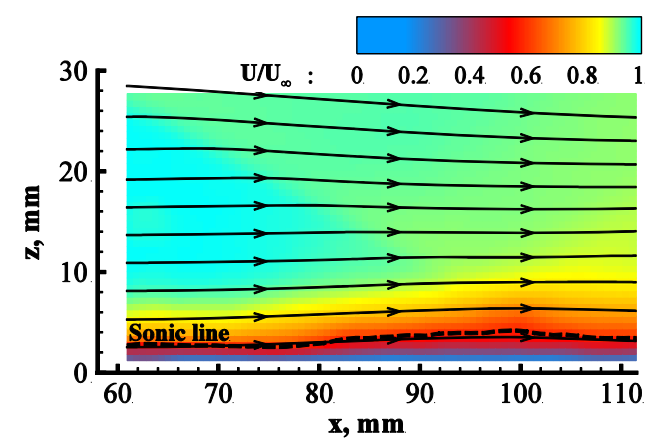

b)

Fig. 8 Ensemble average streamwise velocity field on a) SV1 $(y=28 \mathrm{~mm})$ and b) SV3 $(y=17 \mathrm{~mm})$ planes, respectively. 


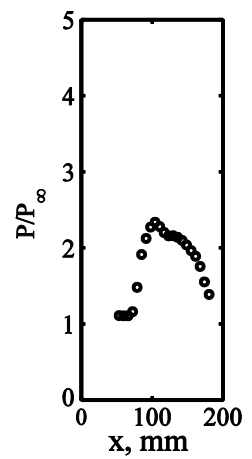

a)

Fig. 9 Average pressure distribution on the a) centerline of the bottom wall and at b) various wall-normal locations on the sidewall $(y=0)$ of the wind tunnel. Pressure data at $z=35.3 \mathrm{~mm}$ have been offset vertically by one unit for clarity.

being a plane of symmetry. On the sidewall, the pressure distribution follows a profile similar to the one on the bottom wall as if the pressure increase due to the interaction is spread equally across the span. The main difference is the presence of a local minimum at about $x=120 \mathrm{~mm}$, which is attributed to the intersection of expansion wave $W, E$ with $C_{2}$. After the interaction region, the pressure distribution decreases again due to expansion wave $E$.
The effect on the flowfield of the sidewall and corner region is better visualized by the set of measurements on selected TV planes, shown in Fig. 10. The selected TV planes span the region immediately upstream and downstream of the nominal interaction region. In particular, Fig. 10 shows planes TV6 through TV11, which cover a region from $15 \mathrm{~mm}(1.5 \delta)$ upstream of I to $10 \mathrm{~mm}(1 \delta)$ downstream of I (see also Fig. 4). In these figures, the white dashed line indicates the sonic line as defined previously, whereas the black solid line indicates the edge of the separated flow $h$, to be defined in the following. The measurement region on these planes approximately spans the lower right corner of the wind-tunnel cross section when looking in the direction of the flow. Plane TV6 (Fig. 10a) is approximately located at the beginning of the region of upstream influence, where the boundary layer near the centerline has not yet thickened (see Fig. 8a). With reference to the earlier Fig. 6, we observe that the flow at the corner has strongly evolved: from just a thickening of the boundary layer at the corner (Fig. 6) into a bulge with a strong velocity defect on the sidewall that protrudes into the core flow. The modification of the corner flow region into what we observe is primarily due to the presence of the incident shock wave interacting with the sidewall boundary layer and the corner flow itself. As the flow evolves downstream, the velocity defect bulge grows deeper into the flow, reaching about one-quarter of the span by TV10. In plane TV7, a lowvelocity region near the tunnel's centerline also begins to appear and grows up to plane TV9, after which it collapses and disappears by TV11. This is the region on the bottom wall affected by the interaction (which does not separate in the mean, as we will assess subsequently), and it is consistent with that shown by the field in

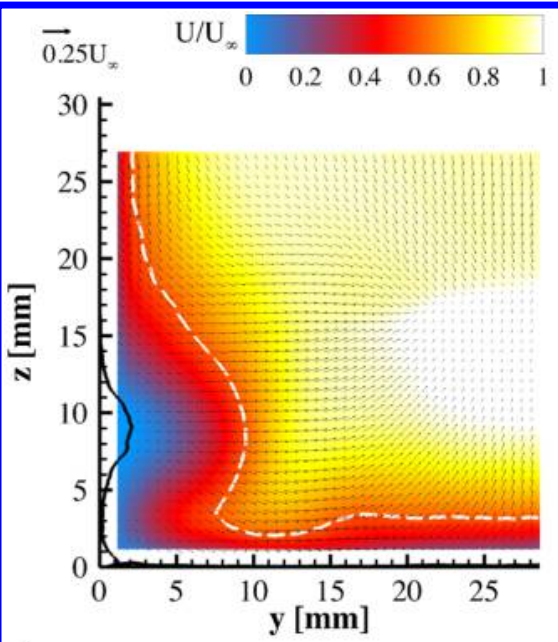

a)

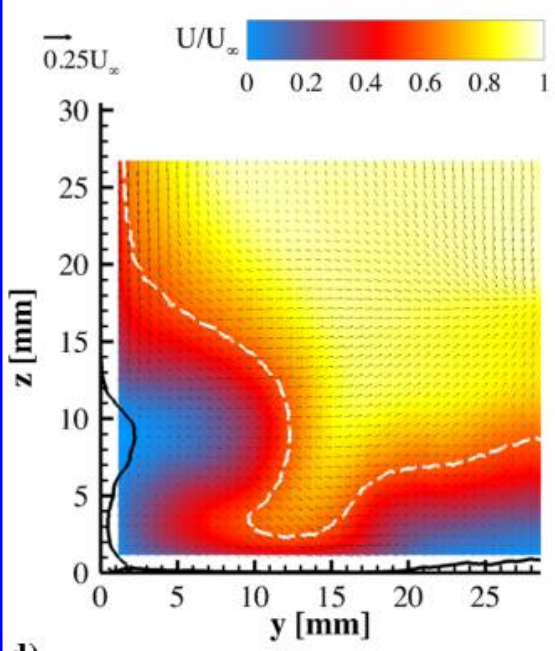

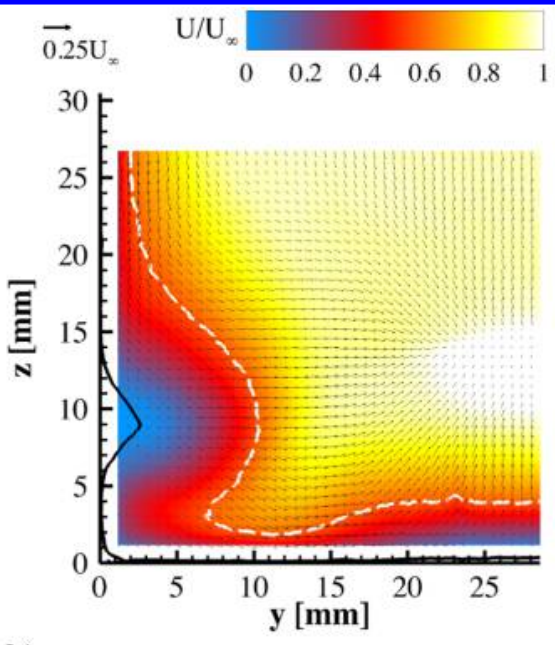

b)

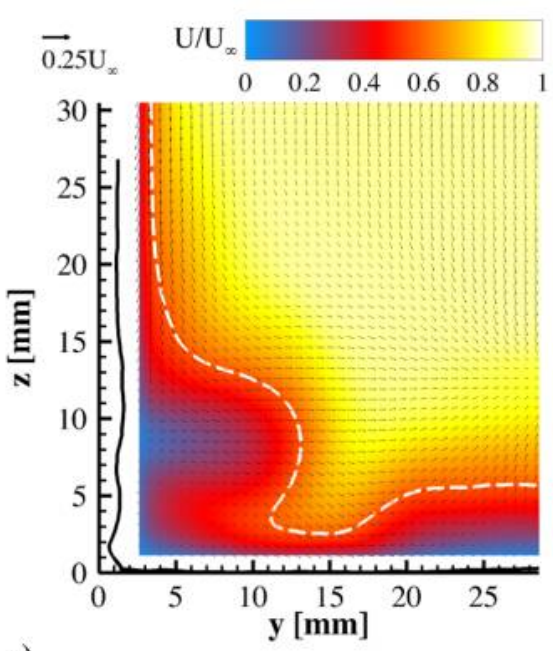

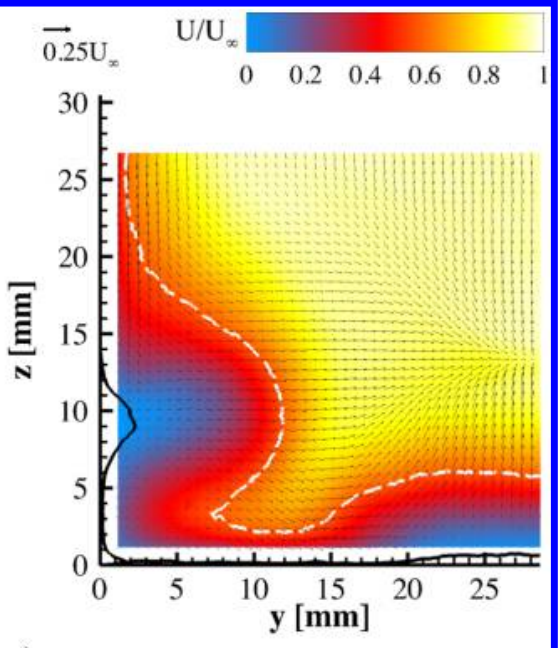

c)

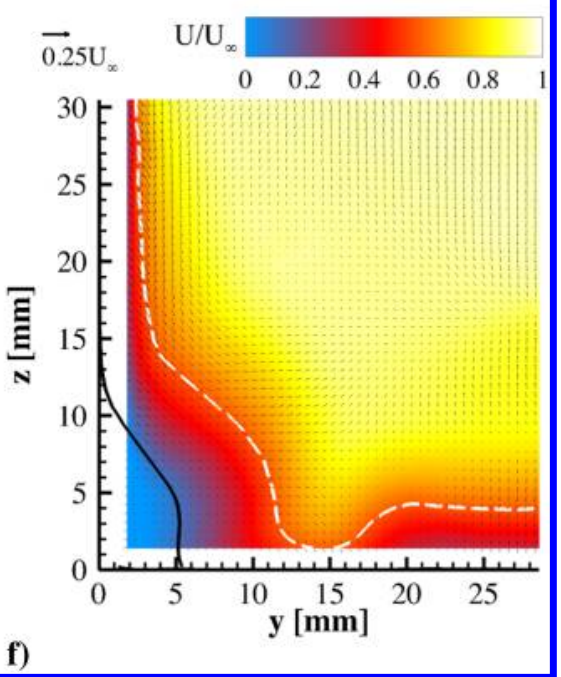


plane SV1 (Fig. 8a). Note also that this region is located only around the centerplane of the tunnel, but it does not reach the sidewall. In fact, it appears to close as the low-velocity bulge near the corner is reached. Thus, at least in the specific case of this study, the bottom wall and sidewall boundary-layer interactions are not directly connected to each other.

To further investigate the flowfield structure in relation to the incident/reflected shocks, the shock structure is extracted from a measure of the in-plane strain inferred from the in-plane spatial gradients that the measurements allow. In particular, the norm of the in-plane strain rate is defined as

$$
\|\boldsymbol{S}\|=\sqrt{S_{y y}^{2}+S_{z z}^{2}+S_{y z}^{2}}
$$

where $S_{y y}=\partial V / \partial y, \quad S_{z z}=\partial W / \partial z, \quad$ and $\quad S_{y z}=0.5(\partial V / \partial z+$ $\partial W / \partial y)$ are the in-plane strain components obtained from the measurements.

Figure 11a shows the norm of the in-plane strain rate on the TV6 plane, with the major shock and flow structure identified by black lines. The incident shock wave is identified as the region of maximum strain. Unlike a truly 2-D interaction, the incident shock is somewhat curved, as has been studied by Alvi and Settles [23], Kubota and Stollery [20], Lu [24], Panaras [25], Wang et al. [38], and Knight et al. [52]; it does not extend to the sidewall but, rather, it dies out at about one-quarter of the span from the sidewall. This is consistent with the previous observation that the bottom-wall interaction extends only up to about one-quarter of the span. The sidewall does not appear to be affected by regions of concentrated large strains. This effect could be attributed to the existence of shock waves. In general, the incident shock exhibits characteristics similar to those shown for plane TV6 (Fig. 11) on TV planes up to plane TV9.

Below the incident shock, we can also observe the formation of the reflected wave induced by the thickening of the boundary layer on the centerplane, which begins at about $x=86 \mathrm{~mm}$. The reflected wave in Fig. 11 would form the upstream part of the incident shock lambda structure reported in many previous studies $[17,19,21]$. In contrast to the incident shock, the reflected wave is highly curved upward and terminates at the incident shock. To the left of the point where the incident and interaction waves meet, the flow is disturbed by the presence of the sidewall and, more importantly, by the low-velocity region (bulge) identified in Fig. 10. Between the incident and reflected shock is the undisturbed flow, which at this location only covers a small area of the full cross section of the wind tunnel (see also Fig. 10a). For example, at this location, if the interaction of the incident shock were 2-D and inviscid, then the region of undisturbed flow would be the region below the $z=18 \mathrm{~mm}$ line, or about five times the size (area) of what we observe in this 3-D interaction. Thus, the strong three-dimensionality of the interaction results in a significantly reduced core cross-sectional area, even at the early stages of the interaction. The general shock structure shown on plane TV6 is also observed for planes upstream of the nominal interaction location, i.e., up to TV8. At plane TV8 (not shown), the incident and reflected waves intersect and cross.

The distortion of the flowfield resulting from confinement effects can also be further visualized by the $v$-and $w$-velocity color contours of Figs. $11 \mathrm{~b}$ and $11 \mathrm{c}$ (superimposed on the shocks identified in Fig. 11a). With reference to Fig. 11b, the presence and evolution of the low-velocity region at the corner induces a strong inward (i.e., from the sidewall toward the centerplane) flow; this inward flow region does not reach the centerplane, but it is bounded by the incident and interaction waves. Similarly, the interaction of the incident shock on the centerplane induces an upflow from the wall into the core flow; the region of upflow is bounded by the bottom wall and the upwardly curved interaction wave. Similarly, the incident shock induces a downflow (bounded by the wedge surface) in the region around the centerplane only. Finally, a strong downflow (about 10\% of the undisturbed streamwise speed) on the sidewall is also present. This region is bounded in a narrow region at the sidewall for $z$ larger than $21 \mathrm{~mm}$ in this case. Although the 2-D strain field computed from the measurements does not reveal the presence of a sidewall shock, we conjecture that this downflow is induced by the presence of a swept-shock-like interaction on the sidewall generated by the incident oblique shock. Many of these wall-normal velocity features were also observed by Helmer et al. [16].

\section{Intermittent Flow Separation and Separated Flow Bubble Height}

The boundary layers on the bottom-walls and side-walls do not separate in the mean but only intermittently. The characteristics of the intermittent separation are investigated by extracting the edge of the separated flow from the set of uncorrelated instantaneous measurements used to construct the ensemble-averaged fields shown previously. Two different methods have been used. In the first method, an intermittency of separation map was constructed by computing the local probability of finding an instantaneous streamwise velocity less than a threshold value indicative of separation [i.e., by defining a probability of reverse flow (PRF)]. Different threshold values ranging from 0 to $60 \mathrm{~m} / \mathrm{s}\left(\sim 0.1 U_{\infty}\right)$ were considered, but all cases showed similar results. Here, we show a case with a threshold value of $25 \mathrm{~m} / \mathrm{s}$, which is about twice the minimum velocity we can measure with our setup and instrumentation. This value was selected to account for limitations of our measurement configuration in measuring low velocities.

In the second method, we use the definition of the separated flow bubble height profile as defined by Piponniau et al. [1] and Souverein et al. [2], who used it to study flows that were attached in the mean but

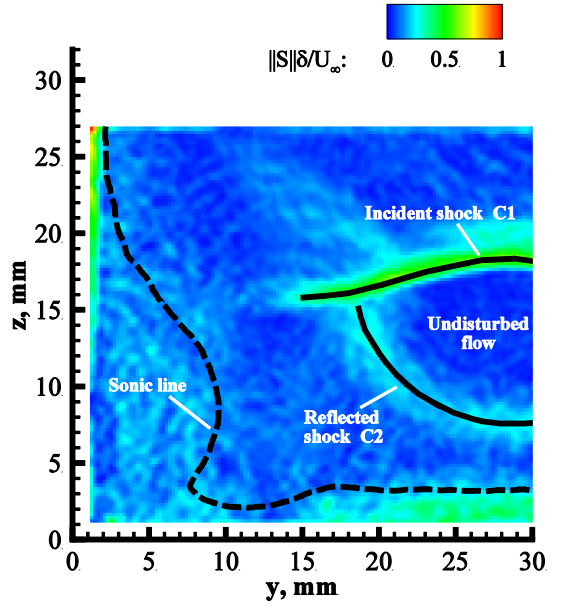

a)

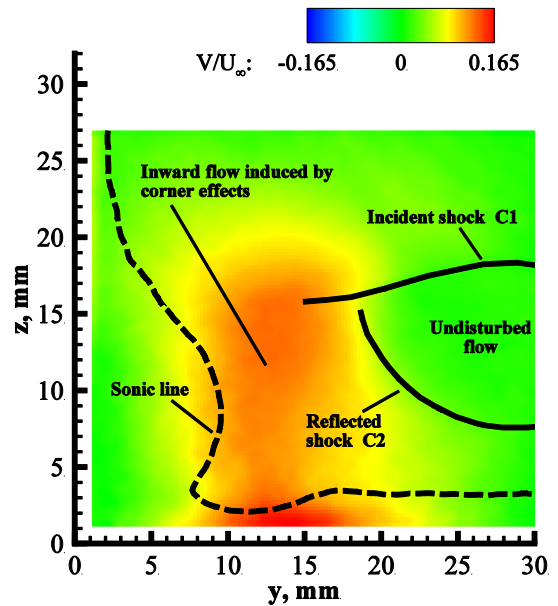

b)

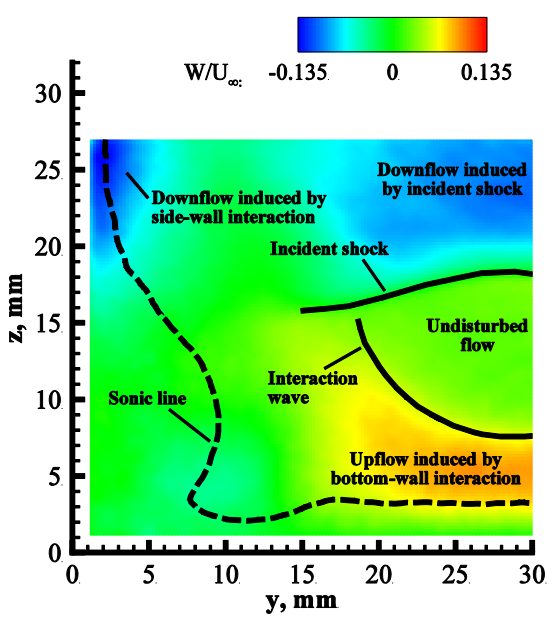

c)

Fig. 11 Average fields at TV6 showing a) planar strain norm $\|S\|$, b), and c) velocity components V, W. 


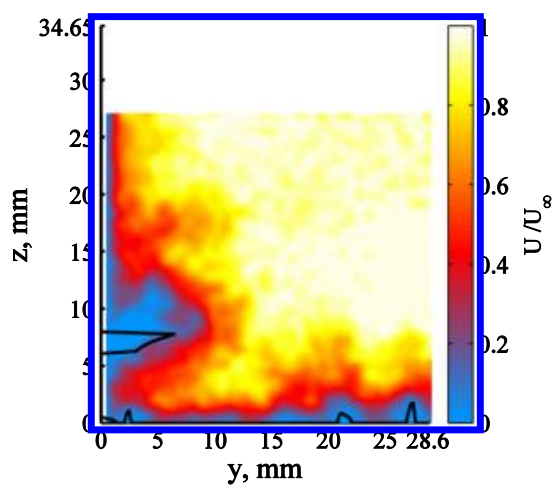

a)

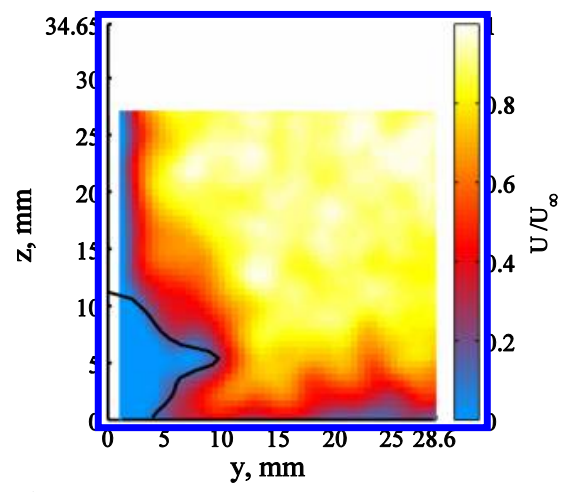

b)

Fig. 12 Instantaneous $U / U_{\infty}$ field superimposed by separation bubble profile at a) TV6 $(x=81 \mathrm{~mm})$, and b) TV11 $(x=107 \mathrm{~mm})$.

were incipiently separated. They defined the separation height profile $h_{k}(t)$ of the $k$ th image as

$$
\int_{0}^{h_{k}(t)} u_{k}(t, n) \cdot \mathrm{d} n=0
$$

where $t$ and $n$ are dummy variables that indicate the direction tangent and normal to the wall contained by the measurement plane (i.e., a TV plane), respectively; e.g., $t=y$ and $n=z$ for the bottom wall, whereas $n=y$ and $t=z$ for the sidewall on a TV plane. The local streamwise velocity at the $(t, n)$ point for the $k$ th realization is $u_{k}(t, n)$. The quantity $h$ is computed for each measurement instant $k$, and it is denoted by $h_{k}$. By definition, if separation is not detected, $h_{k}=0$. The ensemble-averaged quantity, here denoted by $h$, is computed by averaging the set of local values $h_{k}$. This parameter effectively tells us how much of the wall-normal space is effectively reduced because of separation. It is equivalent to the region being unavailable to the incoming flow. It must be noted that the current definition does not include the effect of density; thus, it is an "incompressible flow" equivalent. This quantity is limited by the accuracy of velocity measurements near the wall and by the overall spatial resolution of the measurements. As a result, vector validation, interpolation, and low-pass filtering are performed as described previously.

From each instantaneous $h_{k}$ profile, the maximum value of the profile is extracted and denoted by $H_{k} . H_{k}$ is simply referred to as the separation bubble height and provides a global instantaneous measure of the wall-normal extent of the separated flow. Unlike the quantity $h_{k}$, which is a function of one coordinate direction, $H_{k}$ is a constant for a particular image. The ensemble-averaged separation bubble height $H$ is then computed from the set of instantaneous values. The total area under the $h_{k}$ profile is referred to as the area of separated flow. Finally, we define the local separated flow to pertain to the sidewall if it occurs in a region for $y<10 \mathrm{~mm}$ (i.e., $1 \delta$ ), and it is attributed to the bottom wall otherwise. By this definition, flow separation in the corner region belongs to the sidewall (corner flow separation is not observed to stretch for more than $1 \delta$ away from the sidewall).

TV5 to TV13 planes, which lie around the interaction region, are analyzed for detecting separated flow. Transverse-vertical planes are chosen for this analysis, since they provide for a cross-sectional projection of the separation bubble perpendicular to the core flow direction, which is then used to infer the effective area unavailable to the flow.

Figures $12 \mathrm{a}$ and $12 \mathrm{~b}$ show a representative instantaneous view of the separation bubble height profile $h_{k}$ (black solid line) superimposed on the streamwise (out-of-plane) velocity color contour of planes TV6 ( $1.5 \delta$ upstream of the incident shock impingement) and TV11 ( $1 \delta$ downstream of the incident shock impingement). The first observation to be made is that the region of separated flow on the sidewall is larger than that on the bottom wall, and it is most prominent near the corner of the tunnel. For the location and specific instant shown for plane TV6, the sidewall separation close to the corner is already developed, whereas the bottom-wall boundary layer near the centerplane of the tunnel is not strongly influenced by the incident shock yet. It is apparent from comparing Figs. $12 \mathrm{a}$ and $12 \mathrm{~b}$ that the region of separated flow on the sidewall in TV11 is larger than that inTV6, indicating growth of the separation through the SBLI. This result is also seen in the ensemble-averaged profile $h$ (shown by solid lines in Fig. 10) and from inspection of the probability of reverse-flow maps (shown subsequently in Fig. 13).

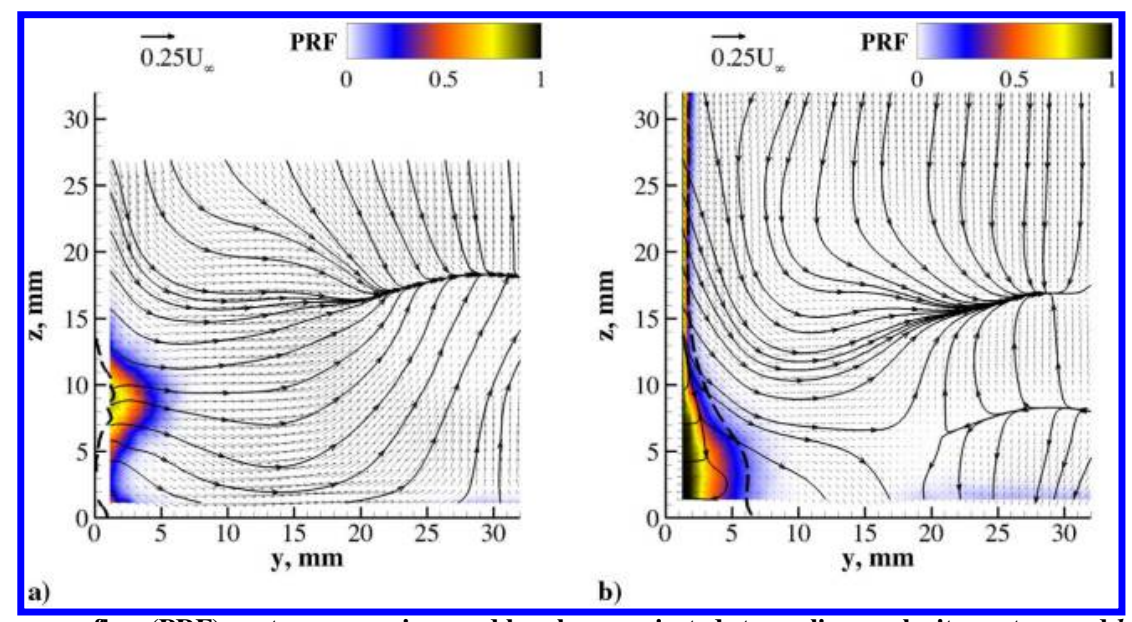

Fig. 13 Probability of reverse flow (PRF) contours superimposed by plane projected streamlines, velocity vectors and $h$ at a) TV6, and b) TV11. 
The ensemble-averaged separation bubble height profiles $h$ corresponding to the planes of Figs. 12a and 12b are indicated in Fig. 10a and $10 \mathrm{f}$ by the solid black line. The ensemble-averaged bottom-wall separation bubble height was found to be negligible (at least within the limitations of the current measurements) and is not visible in the figures, whereas the corresponding profile on the sidewall has a nonnegligible value in the mean. The area under these curves is a representation of the region in the wind tunnel where there is no net flow on average. Comparing Figs. 10a and 10f, flow blockage is

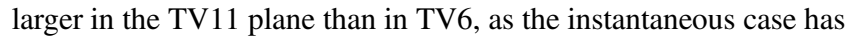
also shown. Most of the contribution to the blockage comes from the sidewall separation in both planes. The bottom-wall separation is nearly nonexistent in the mean at these two locations and only appears in the immediate vicinity of the expected centerline interaction. This behavior can be ascribed to the significant three-dimensional effects arising as a result of the low aspect ratio of the wind tunnel and the complex curved shock structure of the incident shock as the corner flow is approached.

To quantify the likelihood of observing flow separation, the probability of reverse flow for planes TV6 and TV11 is shown on Fig. 13. The figures show the probability superimposed on the mean $h$ profile (thick, black, and dashed line), in-plane velocity vectors, and two-dimensional (projected) streamlines. We observe that, on TV6, the probability of finding separated flow on the bottom wall near the tunnel's centerplane is much less than $50 \%$ (within the measurement region); whereas on the sidewall, we can have flow separation up to $50 \%$ of the time within the region where the lowvelocity bulge identified previously is found. For the TV11 plane, no separation is, in practice, observed on the bottom wall, whereas the sidewall and corner experience flow separation a significant fraction of the time. In the corner region, for example, flow separation approaches $100 \%$. In both cases, the separation bubble height profile $h$ is a good indicator of separated flow and agrees well with the probability map.

Figure 14a shows the probability density function (PDF) distribution of sidewall separated flow area $A$ (normalized by $A_{T}$, which is one-fourth of the cross-sectional area of the tunnel) compiled over all instants for the TV6 and TV11 planes. The results are normalized with one-fourth of the tunnel cross-sectional area, as only the bottom quarter of the tunnel is studied in detail in this work; this choice gives the equivalent fraction if computed over the full cross-sectional area. The ratio $A / A_{T}$ indicates the fraction of the total area blocked to the incoming flow by separation. Flow is considered attached if $A=0$. The upstream plane (TV6) has a smaller sidewall separated flow area, and separation is more intermittent than sidewall separation at the downstream location (TV11), as inferred from Fig. 14a. Comparing Figs. $14 \mathrm{a}$ and $14 \mathrm{~b}$, we conclude that, on the sidewall, the flow state at TV6 is more likely to be attached, whereas the flow is always separated in TV11. On the contrary, the bottom wall is more likely to be attached at both locations. However, note that the finite resolution of the measurements (relative to the boundary-layer thickness and, possibly, to the size of separation that might be present) and practical limitations in measuring the flow velocity as the wall is approached could bias the measurement toward higher velocity, thus preventing correct detection of negative velocities for thin (compared to the size of the PIV interrogation window) reverse-flow regions.

Further downstream, the most probable value of the sidewall separated flow area in the TV11 plane is about $3 \%$ of the quarter-tunnel cross-sectional area $A_{T}$, and there is no case of nonseparated flow, as seen in Fig. 14a. At the most, the sidewall separation on the TV11 plane amounts to up to $6 \%$ of the wind-tunnel quarter-cross-section area.

PDF distributions of the bottom-wall separation area for the TV6 and TV11 planes are shown in Fig. 14b. It is evident that the bottomwall separation is more probable at the upstream location of TV6; although, at both locations, the predominant state is attached flow. The hypothesis put forward by Délery and Dussauge [21] to explain reattachment is that the incident shock wave impinges on the flow around a separation bubble, which acts as a free boundary, causing an expansion wave to be reflected in the downstream direction. The expansion wave curves the detached shear layer toward the wall, eventually reattaching it downstream of the interaction. At the same time, the influence of high pressure developed behind the shock is propagated upstream through the viscous boundary layer. This smears the adverse pressure gradient upstream of the point where the shock impinges the boundary layer on the bottom wall and causes the flow to separate. In Figs. 14a and 14b, it can be seen that most of the flow separation in our flow configuration comes from the interaction of the shock with the sidewall boundary layer and the corner flow, and not from the interaction with the bottom wall.

PDF distributions computed for the total separated flow area (i.e., the sum of the separated area on the side-walls and bottomwalls) from all the images of the TV6 and TV 11 planes are shown in Fig. 14c. For the most part, these PDF distributions follow the profiles for the corresponding sidewall separated flow area. Separation is

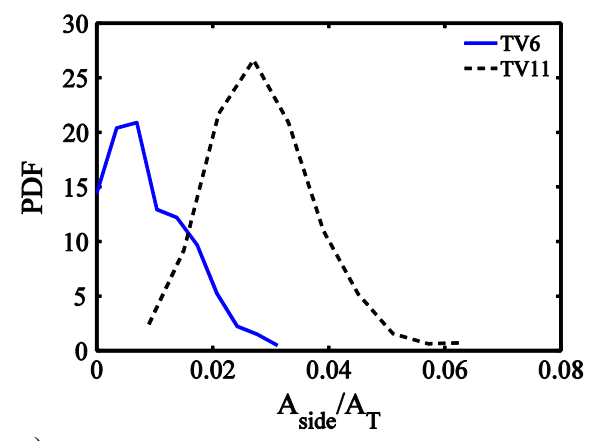

a)

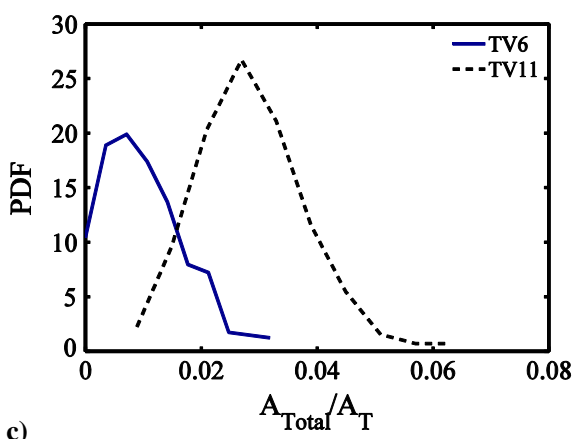

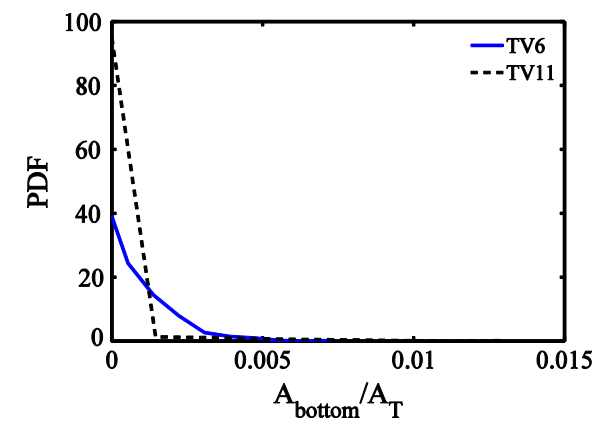

b)

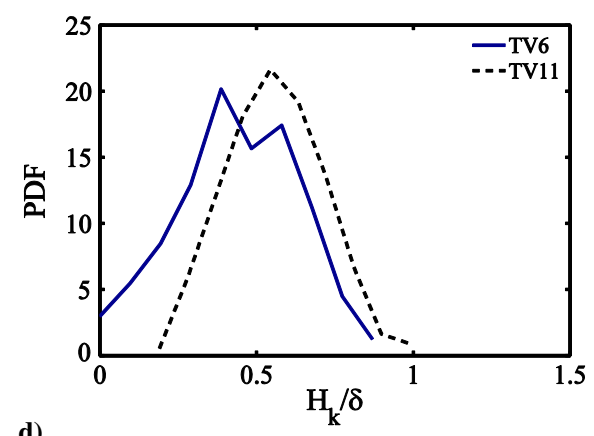

Fig. 14 PDFs of various separation charcteristics at TV6 and TV11 planes. 
more probable and larger in the region downstream of the interaction than the upstream plane.

Figure $14 \mathrm{~d}$ shows the PDF distribution of the sidewall (maximum) separation bubble height $H_{k}$ (normalized by $\delta$ ) for the two planes considered here. This quantity represents how far the region of separated flow stretches into the undisturbed flow. A value of $H_{k}$ equal to zero corresponds to a nonseparated case. It is worth noting that, past the interaction zone, the separation bubble is more likely to stretch toward the core flow than in the upstream region. On the upstream plane, the separation bubble height extends up to $0.5 \delta$; whereas on the downstream plane, it reaches one boundary-layer thickness.

The analysis presented previously was repeated for the planes around the interaction region to obtain the variation of separated flow properties with respect to the streamwise direction $x$. The streamwise variations of the ensemble-averaged total, bottom wall, and sidewall separated flow areas (computed over all instances) are shown in Fig. 15. Since the relevant measurements started at a streamwise location of $x=76 \mathrm{~mm}$, the data are linearly extrapolated to zero (solid lines) to obtain the upstream point of separation, which is found to be around $x \approx 70 \mathrm{~mm}$. It can also be seen that the bottom-wall separation bubble reattaches upstream of the sidewall reattachment at about $x \approx 110 \mathrm{~mm}$. These values are also supported by the static wall pressure measurements made on the bottom-wall centerline, as shown in Fig. 9. It can be seen from the pressure measurements that a sharp adverse pressure gradient is developed at about $x \approx 75 \mathrm{~mm}$, causing the flow to (intermittently) separate, whereas a strong favorable pressure gradient between $x \approx 100$ and $120 \mathrm{~mm}$ causes the flow to reattach on the bottom wall. The static wall pressure measurements on the sidewall follow the same trend of the streamwise variation of separated flow area. In particular, the decrease in the sidewall separated flow area around $x \approx 90 \mathrm{~mm}$ corresponds to the local dip in pressure and the favorable pressure gradient observed about the vertical midplane $(z=34.7 \mathrm{~mm})$, whereas the maximum of the sidewall separated flow area corresponds to the second peak in the pressure plots observed about the vertical midplane $(z=34.7 \mathrm{~mm})$ around $x \approx 110 \mathrm{~mm}$. Note that the local minimum around $x \approx$ $90 \mathrm{~mm}$ is not observed in the pressure plots taken at $z=15.2 \mathrm{~mm}$. The absence of the pressure minimum at this position could be caused by corner effects and the resulting shock system that may tend to smear out pressure variations.

It can be seen from Fig. 15 that most of the contribution to the total separated flow area comes from the sidewall separation. This is particularly true after the nominal interaction. The area of separated flow on the bottom wall grows from $x=70 \mathrm{~mm}$, peaks around the nominal interaction location $(x \approx 96 \mathrm{~mm})$, and then decreases to zero immediately after the interaction. On the other hand, the sidewall separated flow area increases above the value for the bottom wall in the region leading to the nominal interaction region, drops to a local minimum value around the nominal interaction region, and then further increases after the nominal interaction location. At the nominal interaction location, where separation on the bottom wall is at its strongest, the bottom-wall separated flow area accounts for about $35 \%$ of the total separated flow area. Thus, overall, separation on the sidewall is the largest contribution to areas of separated flow. We note

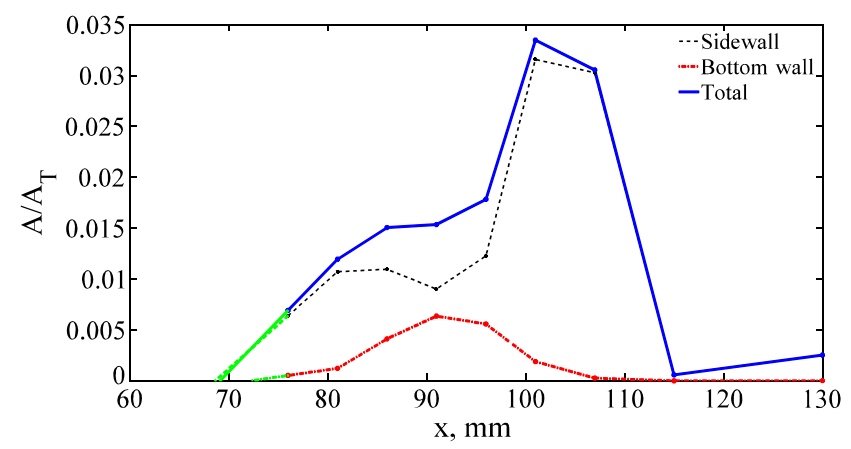

Fig. 15 Streamwise distribution of mean separation area. that, at least for a low-aspect-ratio duct, this result could impact the placement and testing of various boundary-layer control devices and injection systems that have previously been installed primarily on the bottom-walls of supersonic inlet geometries, such as in the studies by Bruce and Babinsky []], Lapsa [39], Lu et al. [53], and McCormick [54], to name a few.

The dominance of separated flow on the sidewall is attributed to the strong three-dimensionality of the flow caused by the SBLI. In the region just before the nominal interaction location, the bottom-wall separation zone strengthens due to the adverse pressure gradient from the shock that is felt upstream, and it may cause the sidewall separation zone to weaken (relative to the incoming state) in the same region. Nevertheless, the contribution of sidewall separated flow to the total separated flow remains greater than the contribution of bottom-wall separated flow. After the nominal interaction location, the bottom-wall separation reattaches due to the expansion fan reflected from the incident shock. But, at this position, the 3-D effects of the interaction associated with sidewall-induced flow deflection toward the center of the duct (and, overall, important because of the low aspect ratio of the tunnel) dominate. As the centerline interaction is diminishing, the separation bubble size on the sidewall continues to grow in the downstream direction. The strengthening of one separation and the weakening of the other seem to indicate the existence of some coupling between sidewall and bottom-wall separated flow regions. Analysis of the separated flow size among instantaneous images did not yield any correlation between sizes, however. Another behavior worth noticing from the extrapolated curves is that the sidewall separation bubble seems to start much further upstream (around $x \approx 68 \mathrm{~mm}$ ) of the central interaction, whereas the bottomwall separation bubble starts at around $x \approx 72 \mathrm{~mm}$. It is postulated that this result may be caused by the shock being curved upstream toward the side-walls, along with viscous effects introduced by the sidewall boundary layer that tend to smear the pressure rise further upstream, thus inducing flow separation on the sidewall earlier than on the bottom wall.

\section{Vortical Features}

To better identify vortical structures, we compute from the available velocity measurement a quantity called the rigid-body rotation vorticity $\omega_{\mathrm{RR}}$, which is one component of the vorticity field. Basic fluid motion can be categorized into rigid-body rotation, irrotational straining, and pure shearing [55]. The traditional Cauchy-Stokes decomposition of the velocity gradient tensor $\nabla \boldsymbol{u}$ separates the flow into two parts: the rate of strain $\boldsymbol{S}$, and rate of rotation $\boldsymbol{\Omega}$ tensors:

$$
\nabla u=\boldsymbol{S}+\boldsymbol{\Omega}
$$

where the rate of rotation tensor $\Omega$ is related to vorticity $\boldsymbol{\omega}$. However, in this form, we cannot differentiate between vorticity contribution due to pure shear with that due to rigid-body rotation about an axis (swirling). In the flowfield studied here, shear dominates the flowfield, and thus makes rigid-body vorticity detection difficult. Thus, an alternative decomposition used for vortex identification is used. In particular, we choose the triple decomposition of motion method of Kolár [3], from which rigid-body rotation vorticity $\omega_{\mathrm{RR}}$ can then be defined. Other vortex identification strategies have been proposed and used in the literature [56-59]. Here, we use a 2-D surrogate of the TDM because it provides us a representation of rigid-body rotation (vortical features) analogous to other vortex identification schemes (e.g., swirling strength) while preserving the sense of rotation (i.e., sign of the rigid-body vorticity), unlike other methods. In the following is a brief summary of the TDM, adapted to a 2-D field to reflect the planar nature of the measurements, taken from the original work of Kolaŕ [3]. It has to be emphasized that the TDM applied here is a planar (2-D) surrogate of the full 3-D form.

\section{Planar Surrogate of Triple Decomposition of Motion}

Triple decomposition allows one to decompose the flowfield motion into an irrotational straining rigid-body rotation and a pure shearing motion. Thus, it allows one to extract the rigid rotation 
component, which is used here to identify vortical flow structures. The decomposition into these three components is effective in identifying rigid rotation only in a particular frame of reference in which pure shear is maximum. The TDM method first requires identifying this particular frame of reference, and only then extracting rigid rotation.

Consider the 2-D surrogate of the true 3-D velocity gradient tensor defined on a TV plane as

$$
\nabla u=\left[\begin{array}{cc}
v_{y} & v_{z} \\
w_{y} & w_{z}
\end{array}\right]
$$

which can be computed from the gradient components available from the planar measurements on our TV planes. The term ()$_{j}$ indicates the derivative along direction $j$ for any quantity. We can apply the rotation operator $\boldsymbol{Q}_{\alpha}$ to $\nabla u$ to introduce an arbitrary rotation by an amount $\alpha$ to the laboratory frame of reference, and we obtain the velocity gradient tensor in the rotated frame of reference $\nabla u^{\prime}$ :

$$
\boldsymbol{Q}_{\alpha} \nabla u \boldsymbol{Q}_{\alpha}^{T}=\boldsymbol{Q}_{\alpha} \boldsymbol{S} \boldsymbol{Q}_{\alpha}^{T}+\boldsymbol{Q}_{\alpha} \boldsymbol{\Omega} \boldsymbol{Q}_{\alpha}^{T}
$$

The rotation operator is defined as

$$
\boldsymbol{Q}_{\alpha}=\left[\begin{array}{cc}
\cos \alpha & -\sin \alpha \\
\sin \alpha & \cos \alpha
\end{array}\right]
$$

Equation (4) can also be simply written as

$$
\nabla u^{\prime}=\boldsymbol{S}^{\prime}+\mathbf{\Omega}^{\prime}
$$

where $\boldsymbol{S}^{\prime}$ and $\boldsymbol{\Omega}^{\prime}$ are the rate of strain and rate of rotation tensors in the rotated frame of reference obtained from applying the CauchyStokes decomposition to $\nabla u^{\prime}$. We can then find the particular frame of reference rotated by an angle $\alpha=\theta$ such that pure shear in this rotated frame of reference is maximum. It can be shown [3] that this condition corresponds to finding the angle $\theta$ such that $\left|\bar{S}_{12}^{\prime} \mathbf{\Omega}_{12}^{\prime}\right|$ is maximum, or simply

$$
\theta=\left\{\alpha: \frac{\mathrm{d}}{\mathrm{d} \alpha}\left|\boldsymbol{S}_{12}^{\prime} \mathbf{\Omega}_{12}^{\prime}\right|=0\right\}
$$

In this particular rotated frame of reference, the velocity gradient tensor can be decomposed in two terms (tensors): a pure shear tensor $\boldsymbol{H}$ (which is maximum), and a term that is referred [3] to as the residual tensor $\boldsymbol{R}$ :

$$
\boldsymbol{Q}_{\theta} \nabla u \boldsymbol{Q}_{\theta}^{T}=\boldsymbol{R}+\boldsymbol{H}
$$

The residual tensor $R$ in this frame contains motion associated only with rigid-body rotation and irrotational straining. It can either be a symmetric or an antisymmetric tensor. For the 2-D case, the residual tensor is given by

$$
\boldsymbol{R}=\left[\begin{array}{cc}
v_{y}^{\prime} & \operatorname{sign}\left(v_{z}^{\prime}\right) \min \left(\left|w_{y}^{\prime}\right|,\left|v_{z}^{\prime}\right|\right) \\
\operatorname{sign}\left(w_{y}^{\prime}\right) \min \left(\left|w_{y}^{\prime}\right|,\left|v_{z}^{\prime}\right|\right) & w_{y}^{\prime}
\end{array}\right]
$$

where the primed terms are the components of $\nabla u^{\prime}$ that are known once $\theta$ has been found.

Once the residual tensor is known, it is rotated back to the original laboratory frame of reference where it is decomposed further into pure strain $\hat{\boldsymbol{S}}$ (symmetric part) and pure rotation $\hat{\boldsymbol{\Omega}}$ (antisymmetric part) using the Cauchy-Stokes decomposition:

$$
\boldsymbol{Q}_{-\theta} \boldsymbol{R} \boldsymbol{Q}_{-\theta}^{T}=\hat{\boldsymbol{S}}+\hat{\mathbf{\Omega}}
$$

Finally, the cross-diagonal term of the pure rotation tensor $\hat{\mathbf{\Omega}}$ gives the $x$ component of rigid-body rotation vorticity, which we define as $\omega_{\mathrm{RR}, x}$. This quantity is used to mark the vortical flow features in our flow.
Note that the definition of solid-body rotation vorticity $\omega_{\mathrm{RR}}$ [Eq. (6)] is similar to that of vorticity $\omega$ [Eq. (3)], but vorticity is defined directly from $\nabla u$ (through $\mathbf{\Omega}$ ), whereas rigid-body rotation vorticity is defined from $\nabla u$ after the pure shear component is separated from it using the TDM method (i.e, from $\hat{\boldsymbol{\Omega}}$ ). Thus, the rigid-body rotation vorticity computed by this method is different than the corresponding vorticity computed directly from $\nabla u$, i. e., $\omega_{\mathrm{RR}, x} \neq \omega_{x}$.

Although the description of the method is given for the specific case of measurements on a TV plane, it can be applied to any other plane. The method was applied to all the mean TV planes to obtain $\omega_{\mathrm{RR}, x}$, the mean SV planes to obtain $\omega_{\mathrm{RR}, y}$, and the mean $\mathrm{SH}$ planes to obtain $\omega_{\mathrm{RR}, z}$, respectively.

\section{Identification of Vortical Features}

A few examples of the rigid-body rotation vorticity $\omega_{\mathrm{RR}, x}$, as defined in Eq. (6) through the TDM approach outlined in the previous section, are shown in Fig. 16 for TV planes TV5, TV9, TV12, TV13, and TV14. The TV5 data plane (Fig. 16a) was chosen, as it offers a clear view of the incoming vortices. At this location, the flow is characterized by three primary vortices. Vortices $A$ and $B$ correspond to a counter-rotating corner vortex pair. Vortex $A$ rotates counterclockwise (positive vorticity), whereas vortex $B$ rotates clockwise (negative vorticity). These are consistent with the findings of Davis Gessner [60], who observed a similar vortex pair. The geometry of the vortices in our case is skewed because the wind-tunnel nozzle is contoured only on the top side. Vortex $\mathrm{C}$ is caused by the swept-shock interaction with the sidewall boundary layer. A description of the properties of a swept-shock/boundary-layer interaction can be found in the work by Alvi and Settles [23]. As the flow evolves downstream, it is observed that, as the incident shock wave approaches the bottom wall, the clockwise rotating vortex $B$ is constrained by the corner and its magnitude increases.

Figure 16d shows that, as the flow further develops downstream, vortex $C$, which is associated with the swept shock, is reflected upward from the corner vortex $B$, and it is then further deflected upward by the reflected shock. From Figs. $16 \mathrm{~d}$ and $16 \mathrm{e}$, it appears that the expansion $W$ deflects the vortex, which then strengthens and is convected downward by the stronger expansion fan $E$ that follows. The results of Fig. 16 also show that the corner vortex $A$ moves toward the center, whereas vortex $B$ is strengthened and remains near the corner. The bending of vortex $A$ away from the sidewall would divert the core flow away from it, which is consistent with the oilflow visualization and the surface streamlines described by Burton and Babinsky [26] and Benek et al. [13].

Figure $1 \overline{7}$ shows the $z$-vorticity contour map on the SH1 plane $(z=9.5 \overline{\mathrm{mm}})$. The negative vorticity toward the sidewall is the projection of the vorticity associated with the incoming swept shock (vortex $C$ ) on the streamwise/horizontal plane. The close proximity of this vortex to the bottom wall is thought to be responsible for the increased sidewall separation in the corner region.

A fourth vortex, labeled $D$, is identified on plane TV12 and the following planes. This vortex is seen to persist downstream and evolve while remaining close to the bottom wall. To better identify the origin of this vortex, we compare it with the vorticity field extracted on other planes. In particular, Fig. 18a shows rigid-body rotation $y$ vorticity on plane SV1, and we can identify a strong region of concentrated vorticity (indicated by $D_{o}$ in Fig. 18) about $1 \delta$ downstream of the nominal interaction region. This region of concentrated vorticity is not associated with the (intermittent) separation on centerline, but it is associated with the flow passing through the interaction above the region of separated flow. As shown in Fig. 12, the height of the region of separated flow extends, at most, up to $z=1.7 \mathrm{~mm}$, whereas the region of intense vorticity is centered around $y=5.7 \mathrm{~mm}$ and extends outward up to $y=8.6 \mathrm{~mm}$ while remaining within the boundary layer. We speculate that vortex $D$ and vortex $D_{o}$ observed on SV1 are somehow related. Specifically, our assessment suggests that they are the same vortex that forms near the centerline of the duct as a spanwise vortex (positive $y$ vorticity), and it is then tilted symmetrically with respect to the duct centerline to aligned itself in the direction of the flow downstream to form a counter-rotating vortex 
pair. What we observe in Fig. 18 is the cross section of the vortex projected on the symmetry plane as the vortex is formed at the interaction, whereas Fig. 16e captures both legs (feature $D$ and $D^{\prime}$ ) of the deflected and tilted vortex on a cross-sectional plane. Note that measurements on this plane are full span and capture the counterrotating nature of the vortex pair $D-D^{\prime}$. (Note also the near symmetry of the flowfield and vortex distribution.) The observed alternating negative-positive-negative vortex tubes conforming the bottom-wall would cause alternating separation and reattachment lines, as proposed by Kornilov [61], downstream of the oblique incident SBLI. The overall structure of vortex $D-D^{\prime}$ is similar to that of a horseshoe vortex formed around a bluff body in a flow. In this flow, we can relate the intermittent separation bubble on the bottom

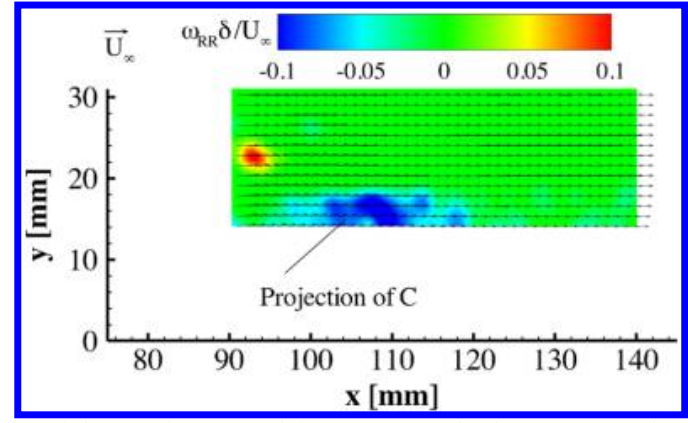

Fig. 17 Rigid rotation $z$-vorticity contours with in-plane velocity vectors on SH1.

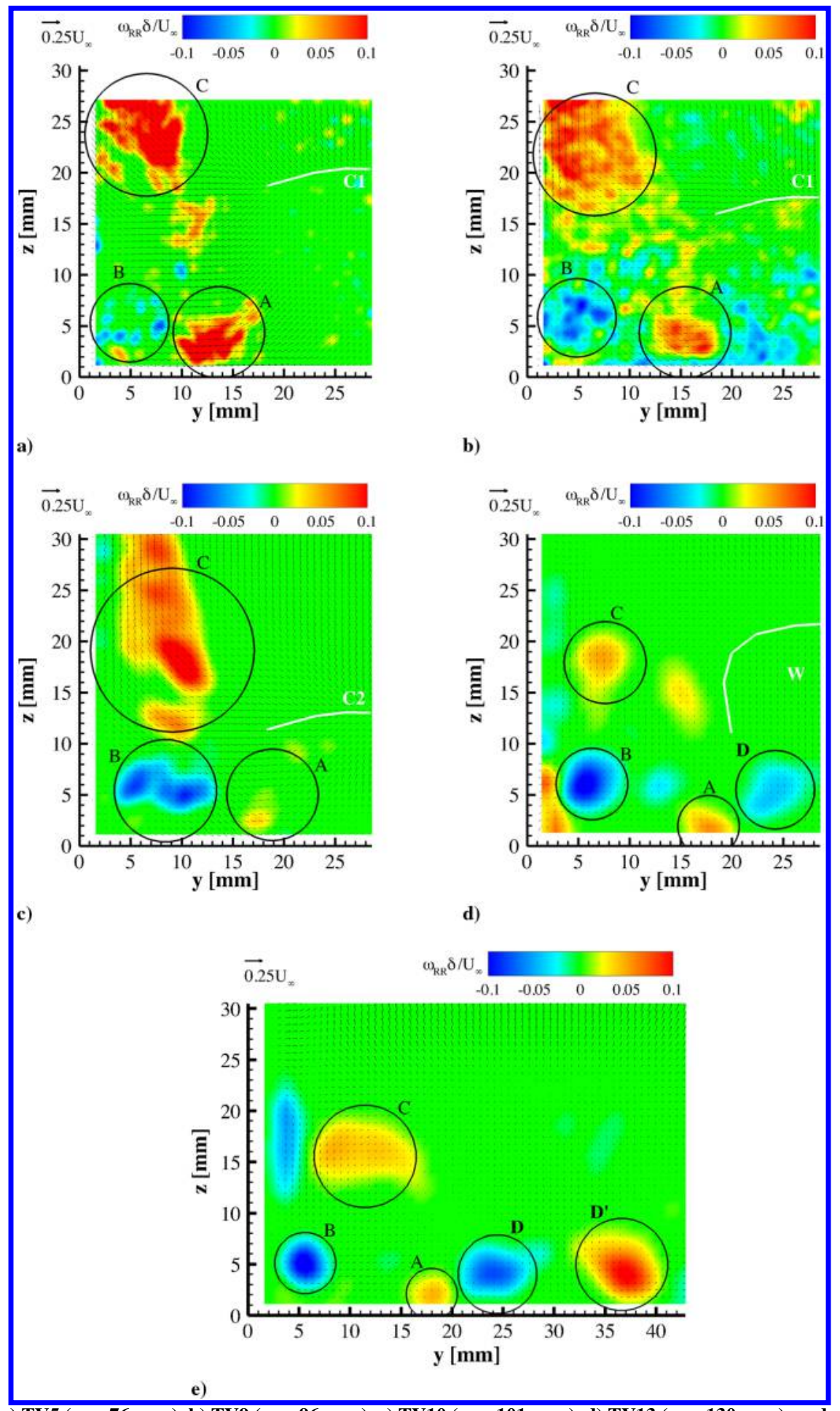

Fig. $16 \omega_{\mathrm{RR}}$ fields at a) TV5 $(x=76 \mathrm{~mm})$, b) TV9 $(x=96 \mathrm{~mm})$, c) TV10 $(x=101 \mathrm{~mm})$, d) TV13 $(x=130 \mathrm{~mm})$, and e) TV14 $(x=137 \mathrm{~mm})$. 


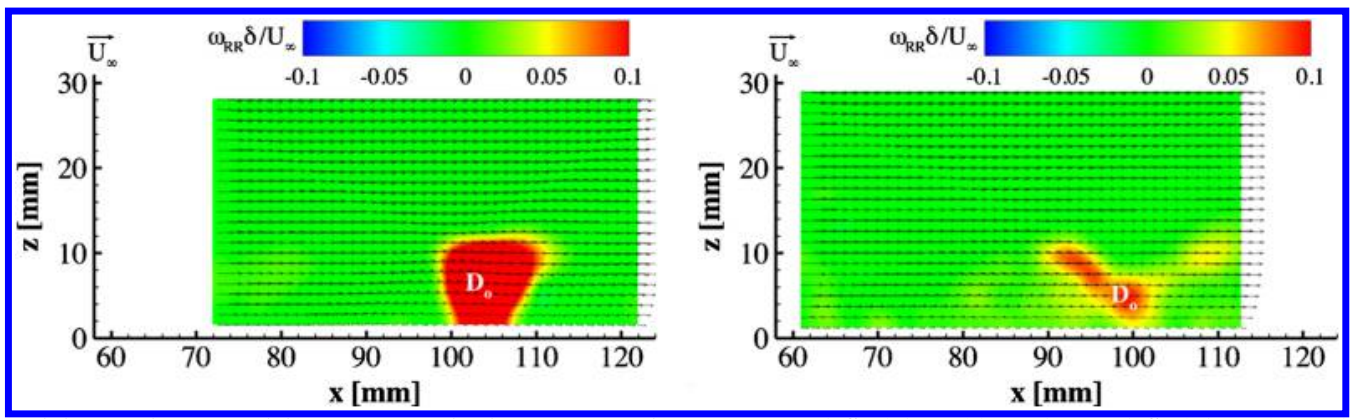

Fig. 18 Rigid rotation $y$-vorticity contours with in-plane velocity vectors on a) SV1 $(y=28 \mathrm{~mm})$ and b) SV3 $(y=17 \mathrm{~mm})$ planes.

wall or even the local thickening of the boundary layer near the centerline of the duct as the bluff-body disturbance that generates the horseshoe vortex we identify in the vortex pair $D-D^{\prime}$. To conclude, note that, from a comparison of consecutive TV planes, we can also observe that the counter-rotating vortex pair $D-D^{\prime}$ tends to move toward the bottom wall because the vortex dynamics drives them downward. As a result, they cause a net downward velocity on the centerline, which may then promote flow reattachment or delay any further separation on the bottom wall.

\section{Discussion}

As can be seen from Fig. 16a, the incoming flow is essentially characterized by two dominant vortex systems: 1 ) the corner vortex pair $A / B$; and 2) the stronger vortex $C$ resulting from the swept SBLI on the sidewall $[52,23]$. Downstream of the interaction region, a third vortex, which we have identified as vortex $D$, becomes important. A schematic diagram that summarizes the possible 3-D structure, distribution, and dependencies of the vortex systems is shown in Fig. 19.

\section{A. Vortex Pair $\boldsymbol{A} / \boldsymbol{B}$}

Vortex pair $A / B$ is associated with secondary flows developing in the corner (described in detail by Gessner et al. [62] and Davis and Gessner [60]) that originate from the full time history of the corner flow forming the flow coming into the interaction. Thus, it is reasonable to assume that the details and response of the vortex pair to a shock may differ from one duct flow to another one. For example, recalling Fig. 6 and the description of the incoming flow properties, the corner flow in our wind tunnel is not symmetric about the corner bisector due to the nonsymmetric nature of our nozzle that induces different pressure gradient and time histories on the developing boundary layer developing on the side and floor of the tunnel merging into the corner [48]. Because the corner vortex pair evolves in a flowfield controlled by two opposing shock interactions on either side of the corner, the evolution of the vortex pair is strongly linked to and responds to the evolution of the other two vortex systems $(C$ and $D)$.

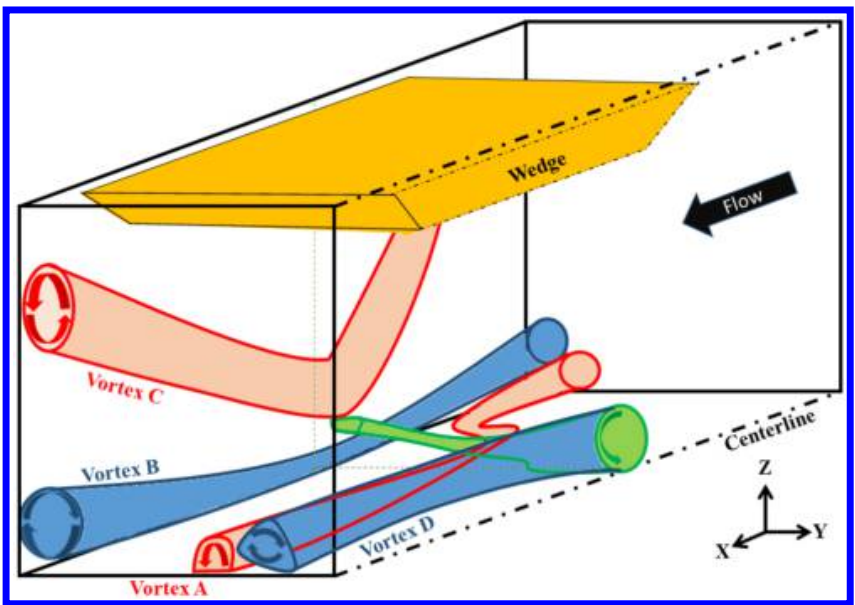

Fig. 19 Schematic diagram of vortex systems structure in the 3-D shock wave/boundary-layer interaction in a duct flow.

\section{B. Vortex $C$}

Vortex $C$ develops on the sidewall and assumes the shape seen in Fig. 16c, which is comparable to what was observed by Panaras [25] in his computations. The curved nature of the incident and reflected shocks is also apparent in Figs. 16a-16c, which is caused by the simultaneous existence of the two coupled shock interaction systems (swept and oblique SBLIs) and the low aspect ratio of the duct. Vortex $C$ is associated with the relatively weak swept-shock interaction on the sidewall that develops as the incident shock approaches the bottom wall and then continues as part of the reflected shock system.

Using the physical model of the free (i.e., not confined by or reflected from walls) swept-shock interaction of Alvi and Settles [23] for a Mach 2.7 and 6 deg flow deflection angle, it would be expected that the interaction results in what they referred to as a "primary separation" case in which flow separation consists of a single flattened spiral vortex. In our case, the interaction is relatively weak compared to the range of cases they studied, but it still falls under the primary separation case as they defined it. A schematic diagram showing the flow structure generated by a swept shock is shown in Fig. 20. It has been adapted starting from the description of Alvi and Settles, with the addition of the confinement of the bottom wall and the other vortex features that resulted from the interaction. Because of the conical symmetry of the swept-shock flowfield, it is more appropriate to describe the flowfield in a plane perpendicular to the incident swept shock and in a conical coordinate system $(\phi, \theta)$. What is shown in Fig. 20 follows this view.

In a swept-shock interaction with primary separation, the pressure increase due to the incident shock lifts the boundary layer $(S$ in Fig. 21) and folds it into the spiral vortex once it reattaches (reattachment $R$ in Fig. 21). The influence of the formation of this vortex propagates upstream to some point $U$. The separation and reattachment lines are conical, and thus is the spiral vortex itself. Because of the conical structure of the flowfield, reverse flow in the laboratory frame of reference (i.e., negative streamwise velocity) might not be expected at all times, especially for weak interactions ("reverse" flow is, however, present in the conical coordinate system and refers to flow moving away from flow coming into the interaction, as seen in the conical coordinate system). In this respect, the conical vortex might be considered a form of open-type separation region [63-66], as discussed by Alvi and Settles [23]. The separation vortex then induces a branching of the incident shock (i.e., the creation of a $\lambda$ foot) with a front and a rear shock wave. In our flow, we identify vortex $C$ as the primary separation conical vortex in the free swept-shock interaction.

In our case, we do not have a free swept-shock interaction because of the presence of the corner and bottom wall. Furthermore, the two other vortex systems interact with vortex $C$, and the swept shock (along with vortex $C$ itself) ultimately reflects from the bottom wall. To reconcile the free swept-shock model with our more complex flowfield, consider the following (refer also to Figs. 19 and 20). Early in the evolution, the incident swept-shock flowfield forms and evolves independently and undisturbed by the constraints imposed by the corner and bottom wall. We observe the presence of vortex $C$ on planes upstream of the interaction close to the sidewall and centered at about $z=20 \mathrm{~mm}$ from the bottom wall (see Fig. 16), but we do not observe reverse flow (in the laboratory frame of reference) at this location (Figs. 10 and 13 ). This would be consistent with vortex 


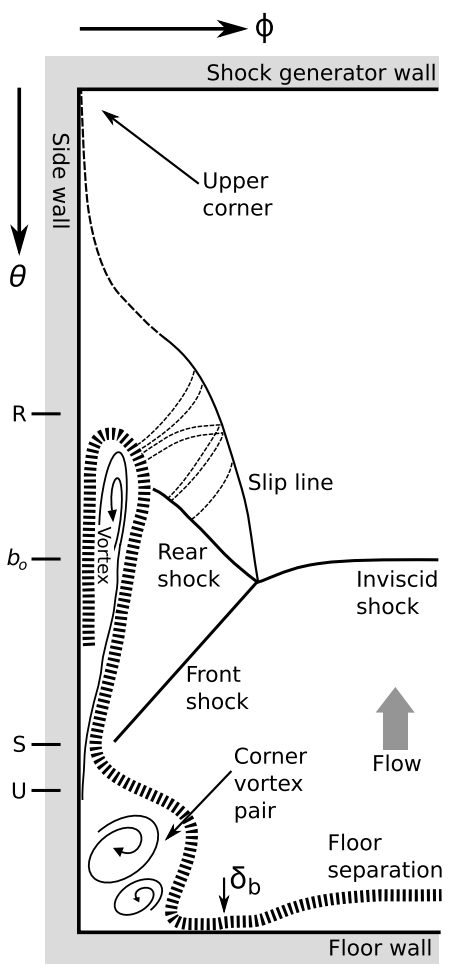

Fig. 20 Schematic SBLI structure on a plane perpendicular to the incident shock.

$C$ being a conical spiral vortex induced by the swept shock on the sidewall and providing a region of open-type separation. Below vortex $C$, we do observe the low streamwise velocity bulge and (intermittent) flow separation in a region close to the sidewall centered at $y=7 \mathrm{~mm}$ (Figs. 9 and 12); but, this feature is associated with the asymmetric corner vortex pair, and its role in weakening the flow at the corner (i.e., by making it more prone to separation) by asymmetrically reorganizing high- (into the lower portion of the corner close to the bottom wall) and low-momentum (away from the corner, in the upper part of it) fluids. As a result, the flow weakly separates in the bulge region upstream of the nominal interaction point away from the bottom wall (Figs. 10a and 13a). Separation in this region is initiated by the adverse pressure gradient associated with the incident shock interaction (see Fig. 9). Then, the flow more strongly separates right at the corner once the incident shock reaches the bottom wall and the corner (Figs. $10 \mathrm{f}$ and $13 \mathrm{~b}$ ). This larger and stronger separation appears to be associated with fluid originating from the region of separated flow starting upstream and being swept down toward the corner vertex. Note that the point of maximum wall pressure is observed near TV11, which is where strong flow separation at the

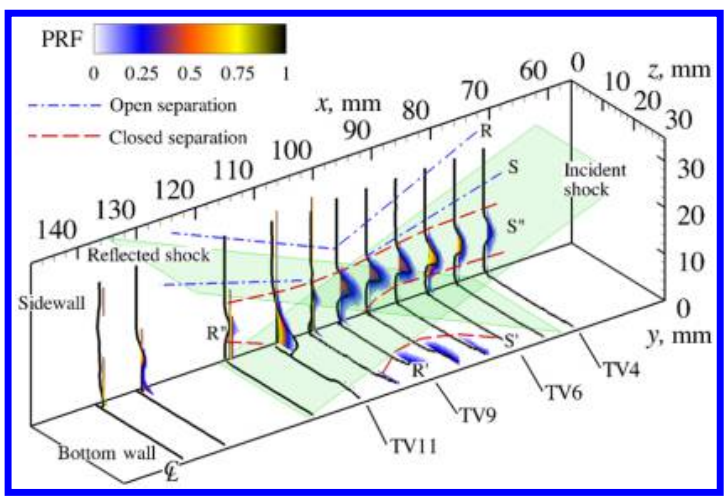

Fig. 21 Three-dimensional plot of PRF and separation bubble height $h$ around the SBLI. corner vertex is observed in Figs. $10 \mathrm{f}$ and $13 \mathrm{~b}$. Note also that this region of separated flow in the corner region is of the closed type (the methods based on defining the separation bubble height profile $h$ and the probability of reverse-flow map, shown in Figs. 10 and 13 , are capable of identifying closed separation only). Thus, vortex $C$ defines a sidewall open separation due to the swept shock, whereas vortex pair $A / B$ indirectly induces a closed separation in the corner itself. Vortex $C$ and the vortex pair $A / B$ mutually interact as the incident shock closes on the bottom-wall corner to create the conditions for generating and sustaining the region of stronger separated flow at the corner vertex.

The interaction between the two vortex systems also affects their trajectory. From the set of vorticity plots in Fig. 16, we can also observe that the trajectory of vortex $C$ is downward toward the bottom wall (see also Fig. 19). As vortex $C$ approaches the bottom wall, it displaces vortex $B$ further down toward the bottom wall, which then drives vortex $A$ toward the centerline of the tunnel, and which is ultimately bounded by vortex $D$ and the plane of symmetry (the centerplane of the duct). Then, as the flow proceeds through the interaction, vortex $C$ begins to follow an upward trajectory and induces a secondary counter-rotating vortex bounded by the sidewall (Fig. 16e). Thus, this vortex dominates the vortex system associated with the reflected shock wave near the corner (Fig. 16d).

\section{Vortex $D$ and Vortex Branching}

The first two vortex systems discussed so far are associated with the swept-shock interaction of the incident shock on the sidewall, the presence of the corner, and the corner vortex pair. As the incident shock finally reaches the bottom-wall boundary layer, a third vortex system is formed. A signature of this third vortex system was identified by vortex $D$ in Fig. 16 and vortex $D_{o}$ in Fig. 18 in both SV1 and SV3. We also observe that vortex $D_{o}$ appears to split in SV3. Because of the somewhat limited set of measurements, we do not have a complete view of the origin of this third vortex system. However, starting from what we discussed so far, we here attempt to reason about its origin and the overall structure from the available data.

Vortex $D$ appears to be relatively decoupled from the flow evolution on the sidewall and at the corner, although its presence may limit the evolution of the vortex pair $A / B$, which tends to be displaced outward toward the centerplane by vortex $C$. Combining the information inferred from Figs. 16 and 18 about vortex $D$ in the previous section, we speculate that vortex $\bar{D}$ may be part of a horseshoe vortex system generated by the boundary-layer thickening or separation near the centerline of the tunnel. However, even this interpretation might be incomplete.

Previous work in our tunnel by Eagle et al. [30] focused on the study of the topology of the flowfield primarily through oilflow visualization. That work identified some of the vortex features studied in more detail here, but it also identified a region of recirculation on the sidewall near the bottom-wall interaction point. This region was associated with a focus point existing on the sidewall, and it was observed that the size of the swirling region increased with the strength of the interaction (i.e., with increasing flow deflection angle of the shock generator). Its origin was not clear, but we currently have two hypotheses about its relation to the vortex $D$ system.

The first hypothesis is that the swirling observed at the sidewall is associated with the closed recirculation region observed at the corner, and it is solely a result of the corner flow; whereas vortex $D$ is the horseshoe vortex described previously. If this were true, we would expect that a similar recirculation region exists on the bottom wall at a corresponding position. Thus, the closed recirculation region would have a shape of a toroidal sector that terminates on the bottom-walls and side-walls, with one focus on either wall. Unfortunately, the oilflow visualization does not show the existence of a second focus point on the bottom wall (may be due to unsteadiness or intermittency of the flow), nor do we have stereo-PIV measurements available close to and parallel to the bottom wall that could provide more insight on this possibility. 
The second hypothesis we consider is that the focus point on the sidewall observed from the oilflow visualization, the vortex $D_{o}$ in $\mathrm{SV} 1$ and SV3, and vortex $D$ are somehow linked, as schematically shown in Fig. 19 through a vortex branching process. The hypothesis is that a vortex spans the full width of the duct and it is anchored on the sidewall; whereas a symmetric U-shaped branching occurs somewhere between the centerplane of the duct and the side-walls to form vortex $D$, which we observe on TV planes. The direction of rotation of the vortex pair $D-D^{\prime}$ observed in TV planes is consistent with this hypothesis. The vortex $D_{o}$ spanning the bottom wall along the $y$ direction observed in the SV planes has a positive $y$ vorticity. If this vortex tube were turned by $90 \mathrm{deg}$ in the positive $z$ direction of rotation, it would have a rotation in the negative $x$ vorticity, which is consistent with the sign of rotation of vortex $D$ in the TV planes. Similarly, the sign of rotation of vortex $D^{\prime}$ is consistent with the turning of vortex $D_{o}$ by $90 \mathrm{deg}$ in the negative $z$ direction of rotation.

Spanwise vortices associated with flows past finite-span bluff bodies attached to a flat surface are widely observed and discussed in the literature $[67,68]$. In our case, a streamwise adverse pressure gradient exists at the nominal interaction region. Pressure is, however, nearly uniform across the span (refer back to Fig. 8 ); thus, the spanwise vortex can terminate on the sidewall and it does not spill over the sides, as in the case of flow around finite-span bluff bodies [67,68].

The studies of Tropea and Martinuzzi [67] and Chou and Chao [68] showed that the spanwise vortex formed at the bluff body could branch into a number of horseshoes $U$ vortices as the ratio between the length $L$ of the bluff body causing the vortex to its height $d$ is increased. In our case $L$ corresponds to the span of the bottom-wall interaction or width of the tunnel and $d$ corresponds to the thickened bottom-wall boundary layer as a result of the interaction which would act similar to a bluff body for the incoming flow. We define this ratio as the slenderness ratio, $L^{*}=L / d$, and we refer to this process as $U$ branching. In our case, we take the length to be the width of the tunnel $\left(L=y_{T}\right)$ and the height of the thickened boundary layer at the interaction on SV1 (specifically, we take the maximum height of the sonic line in Fig. 8a as the representative height of the body "blocking" the flow, and which is approximately on the order of $\delta / 2$ ). Thus, in our case, $L^{*}=y_{T} / d \approx 10$. Chou and Chao [68] considered slenderness ratios ranging from 5 to 20 , and they observed the formation of no branches, one branch, two branches, and three branches as the value of $L^{*}$ increased over this range. The first branching occurred for a slenderness ratio near 10 . Thus, our case might exhibit vortex $U$ branching. Note, however, that their study was performed in water flows at low Reynolds numbers, and our estimate of the size of the equivalent bluff-body size is somewhat arbitrary.

The flow recirculation structure visualized by the oilflow visualization of a stronger interaction (Mach 2.0, 10 deg deflection SBLI; study by Eagle et al. [29]) in the same wind tunnel corresponds to a case of smaller $L^{*}$ (owing to larger representative blockage or $d$ caused by the stronger interaction) and is similar to the structure of the vortex cross section observed by Chou and Chao [68] for a case of $L^{*}=5.33$, where they observed no branching. However, no quantitative data are available from the stronger interaction study [29] to confirm the nonexistence of $D-D^{\prime}$ in the case with a stronger interaction.

\section{Summary of the 3-D Structure of the Vortical Flow and Separated Regions}

Based on the quantitative and qualitative assessment of the different flow properties presented so far, a 3-D schematic diagram showing the distribution of the different vortex systems is presented in Fig. 19. The alternating arrangement of positive and negative vortex tubes conforming to the bottom-wall would be a likely cause of the alternating regions of separation and reattachments observed by Kornilov [61]. Furthermore, a 3-D plot showing the probability of reverse flow (contour), the profiles of separation bubble height $h$ (black solid line), and a schematic representation of the various separation $\left(S, S^{\prime}\right.$, and $\left.S^{\prime \prime}\right)$ and reattachment $\left(R, R^{\prime}\right.$, and $\left.R^{\prime \prime}\right)$ lines are shown in Fig. 21. For clarity, only the TV planes were used to construct Fig. 21. Open-type (dashed-dotted lines) and closed-type dashed lines) separations are differentiated by the line type. For closed separation, the separation and reattachment lines are defined as qualitatively bounding regions of significant probability of reverse flow. The region between the $\mathrm{S}$ and $\mathrm{R}$ lines are only qualitatively located to represent the location of the sidewall vortex induced by the swept shock, which we associate to an open separation consistent with the primary separation flow structure of a weak swept-shock interaction (shown in Fig. 20). Neither the probability of reverse flow nor the separation bubble height methods are capable of identifying this open separation region because these quantities are defined on the detection of reverse flow, whereas close separation does not involve reverse flow [66]. The planes are a qualitative representation (neglecting shock curvature) of the incident and reflected shock wave (angles extracted from the schlieren image of Fig. 5).

The region of closed separation associated with the bottom-wall interaction is confined in a relatively small region on the bottom wall. Its streamwise extent $L_{\text {sep.BW }}$ is only about $2 \delta$ long (somewhere between TV 6 and TV10), extends laterally to about $\pm \delta$ from the duct centerplane, and is about $1 / 3 \delta$ high at most. Separation on the bottom wall is only intermittent, and the probability of observing reverse flow is only $25 \%$.

The closed separation at the corner starts upstream of TV4 and terminates past TV12, which corresponds to a streamwise extent $L_{\text {sep,SW }}$ of at least $\sim 5 \delta$ and penetrates into the duct for about $0.8 \delta$, whereas it remains confined on the sidewall but off the corner itself until the reattachment shock is formed. The most upstream point is where flow reversal is at about $2.5 \delta$ upstream of the nominal interaction point (TV4) and shows a probability of reverse flow around $25 \%$. The probability increases as the flow evolves in the corner, and it reaches a value near 75\% at the interaction itself (around TV9). Up to the interaction point, separation is only intermittent however. Completely separated flow (i.e., $100 \%$ probability of finding reverse flow) is found only $1 \delta$ downstream of the interaction and is observed right at the corner of the duct. The region of separated flow then slowly closes downstream at a position about $2 \delta$ downstream of the interaction (past TV12). Thus, the sidewall region is characterized by a larger region of more likely separated flow than the bottom-wall region. In this sense, the sidewall interaction is said to be the dominant feature.

Recently Bruce et al. [12] and Benek et al. [13] investigated the effects of corners, side-walls, and confinement effects on the different shock wave/boundary-layer interaction configurations. Specifically related to this work, the recent computational work of Benek et al. [13] was aimed at investigating the effect of the duct aspect ratio $\left(\overline{y_{T}} / z_{T}\right)$ and boundary-layer thickness on the regions of separated flow induced by different-strength incident SBLI cases. Their results showed that, independent of the strength of the shock and duct aspect ratio, the boundary-layer thickness-to-duct-width ratio $\left(\delta / y_{T}\right)$ had a primary role in determining the balance between the size and shape of the bottom wall and sidewall regions of separated flow and the overall structure of the vortical flows. In particular, their parametric study showed that, as $\delta / y_{T}$ increased, the overall morphology of the interaction and separated flow regions was strongly affected. More significantly, they found a nonmonotonic trend between the bottomwall separation length and $\delta / y_{T}$ : there existed an intermediate value of $\delta / y_{T}$ at which $L_{\text {sep,Bw}} / \delta$ was maximum. A phenomenological explanation of this behavior was that, for low values of $\delta / y_{T}$, the two interactions developed in a decoupled fashion (effectively, one being a 2-D incident SBLI, and the other a free swept SBLI); as $\delta / y_{T}$ increased, the two interactions coupled to affect each other and to limit their development. But, the dominant flow mechanism controlling the coupling was primarily the region at the corner, and not the sidewall interaction itself. For sufficiently large $\delta / y_{T}$, the development (growth) of the bottom-wall separation was effectively hindered by the constraining of the sidewall separation at the corner (not at the swept SBLI itself), which now dominated the domain with large recirculation regions and vortical structures. The swept shock might have had a role by sweeping sidewall boundary-layer fluid toward the corner. Furthermore, as a result of the displacement of the sidewall flow toward the center, the bottom-wall separation size was reduced (in both the lateral and streamwise extents). The dominance of the sidewall/corner separated flow over the bottom-wall centerline 
separation was enabled by the fact that the footprint of the interaction was convex (i.e., the influence of the bottom-wall interaction was more upstream as the side-wall/corner was approached): the corner flow began to develop undisturbed upstream of the centerline separation, whereas the centerline bottom-wall separation developed in the "wake" of the corner flow separation.

Although our study considers only one interaction strength (freestream Mach number and shock deflection angle) and one case of aspect ratio $\left(y_{T} / z_{T}=0.83, \delta / y_{T}=0.17\right)$, it supports the general observations of Benek et al. [13] on the dominance of the sidewall and corner interactions in low-aspect-ratio supersonic duct flow: although, quantitatively, the response of different flow properties might be somewhat different from theirs.

\section{Conclusions}

In this work, the three-dimensionality of the properties and flow structure of a shock wave/boundary-layer interaction in a low-aspectratio supersonic duct flow was investigated. Stereoscopic-PIV measurements on several orthogonal planes around the interaction regions were used for the study. Measurements on cross-sectional planes were of particular significance for understanding the properties and the coupling between the bottom-wall (incident SBLI) and sidewall (swept SBLI) interactions. It was observed that the incident shock was deformed (curved) toward the core flow, which was in agreement with the simulations of Wang et al. [38]. Because the interaction was relatively weak (6 deg deflection angle), only intermittent separation was observed, which agreed with the findings from some of the cases of Souverein et al. [2]. From the data of Figs. 10 and 21 on TV planes, it was found that the largest velocity deficit and the highest likelihood of separation occurred near the corner region. The topology and location of these regions were different from those observed in the oilflow experiments by Bruce et al. $[12,34]$ and Burton and Babinsky [26]; the primary reason for this difference would likely be the swept-shock interaction present on the sidewall in this experiment. Three-dimensional representations of the areas of flow separation (Fig. 21) and of vortical features (Fig. 19) were constructed to identify key features in the flow and their relative location. They revealed the complex 3-D vortical flowfield that coupled that sidewall and bottom-wall interactions, with the corner region being constrained by the vortices and having a larger tendency to separate. Thus, for the current low-aspect-ratio duct flow, it was concluded that the corner flowfield dominated the interaction in the sense that it was the location of the largest and more probable flow separation. The shape of the flow-separation bubble on the bottom wall was similar to that observed in the simulations of BermejoMoreno et al. [9]. The presence of the attached flow region on the bottom wall between the bottom-wall separation and the corner separation was consistent with the results of Wang et al. [38]. Furthermore, it was demonstrated that the (rigid-body rotation) vorticity was affected by the interaction of the incident shock system on the bottom wall and side-walls, and it resulted in a system of vortices on both walls. It is noted, however, that the reconstructed structure of the vortical features was conducted from a two-dimensional surrogate of the velocity gradient tensor, which might put a limit on how these vortical features are identified.

The current flow configuration was highly unsteady with intermittent separation. Similar results for the case of a high Reynolds number and weak incident shock were observed on the bottom wall by Souverein et al. [2]. Conditional statistics were used to identify and quantify the locations, areas, and probabilities of separation. Maps quantifying the local probability of reverse flow and the separation bubble height $h$ along with its statistics were constructed. Three regions of separated flow were identified from these quantities: 1) the bottom-wall centerplane separation; 2) the corner flow separation; and 3) the sidewall interaction and separation. The first two regions were of the closed-type separation, whereas the third region was of the open type. The sidewall flow was controlled by a conical vortex induced by the swept-shock interaction with no reverse flow (in the laboratory frame of reference). Bottom-wall flow (closed-type) separation was present, but the probability of observing it was only about
$25 \%$ on a relatively small region. At most, its length was limited to about $2 \delta$. The three regions were coupled, and their mutual interaction balanced their strength and size, as suggested by the study of Benek et al. [13]. In fact, the ratio of the boundary-layer thickness relative to the duct size was within the range of values for it to play a controlling role on the growth of the bottom-wall separated flow region, as indicated by Benek et al. [13]. A closed separation (up to $75 \%$ probability of observing reverse flow) was also found at the corner on the sidewall of the duct around TV11 (see Figs. 13 and 21). This was the dominant closed separation region. The side wall separation began well upstream $(2.5 \delta)$ than the bottom wall separation and closed well downstream $(2.5 \delta)$ of it. Its location, size, and strength (probability of finding separated flow) were the result of a balance between the competing development of the sidewall shock interaction, the bottom-wall interaction, and the asymmetric weak corner flow generated by the one-sided nozzle.

The underlying 3-D vortex structure was identified by applying the triple decomposition of motion to isolate the rigid-body rotation vorticity from the shear-related one. This decomposition was applied to a 2-D surrogate form of the velocity gradient tensor that was defined based on the components made available by the current measurement configuration while neglecting the effect of compressibility. This approach provided an effective way to identify swirling motion only, as well as to reconstruct the location and 3-D structure of vortical flow features that dominated the interaction. The method identified three systems of vortices: 1 ) the corner vortex pair (vortex $A / B) ; 2$ ) the swept-shock vortex on the sidewall (vortex $C$ ); and 3 ) a horseshoe-like vortex originated from the bottom-wall interaction, and possibly connected to the corner separation through a branching process. The three vortex systems approached each other at the nominal interaction region, and their combined rotational velocities exerted a strong velocity away from the wall, which tended to promote separation, particularly at the sidewall corner region. The bulk of the structure proposed was similar to the structure proposed by Eagle and Driscoll [4]; however, the lifted type-2 vortex pair was not observed in the TDM results, possibly indicating it was a result of shear or straining phenomena. The alternating negative-positive-negative vortex tubes conforming the bottom wall would also reaffirm the propositions of alternating separation and reattachment lines, as proposed by Kornilov [61], downstream of the oblique incident SBLI.

\section{Acknowledgments}

This work is sponsored by the U.S. Air Force Collaborative Center for Aeronautical Sciences, which is supported by the U.S. Air Force Research Laboratory, Air Vehicle Directorate, AFRL/RB and monitored by John (Jack) Benek.

\section{References}

-11] Piponniau, S., Dussauge, J. P., Debiève, J. F., and Dupont, P., “A Simple Model for Low-Frequency Unsteadiness in Shock-Induced Separation," Journal of Fluid Mechanics, Vol. 629, June 2009, pp. 87-108.

-[2] Souverein, L. J., Dupont, P., Debiève, J.-F., Van Oudheusden, B. W., and Scarano, F., "Effect of Interaction Strength on Unsteadiness in ShockWave-Induced Separations," AIAA Journal, Vol. 48, No. 7, July 2010, pp. 1480-1493. doi:10.2514/1.J050093

- [3] Kolář, V., "Vortex Identification: New Requirements and Limitations," International Journal of Heat and Fluid Flow, Vol. 28, Aug. 2007, pp. 638-652. doi:10.1016/j.ijheatfluidflow.2007.03.004

[4] Eagle, W. E., and Driscoll, J. F., "Shock Wave-Boundary Layer Interactions in Rectangular Inlets: Three-Dimensional Separation Topology and Critical Points," Journal of Fluid Mechanics, Vol. 756, Sept. 2014, pp. 328-353.

[5] Eagle, W. E., "An Experimental Study of Three-Dimensional Inlet Shock-Boundary Layer Interactions," Ph.D. Thesis, Univ. of Michigan, Ann Arbor, MI, 2012.

[6] Morajkar, R., Klomparens, R., Eagle, E., Driscoll, J., and Gamba, M., "Flow Separation Associated with 3-D Shock-Boundary Layer Interaction (SBLI)," 52nd Aerospace Sciences Meeting, AIAA Paper 2014$1138,2014$. 
- [7] Korkegi, R. H., "Comparison of Shock-Induced Two- and ThreeDimensional Incipient Turbulent Separation," AIAA Journal, Vol. 13, No. 4, 1975, pp. 534-535. doi: $10.2514 / 3.49750$

-[8] Bruce, P. J. K., and Babinsky, H., "An Experimental Study into the Flow Physics of Three-Dimensional Shock Control Bumps," 49th AIAA Aerospace Sciences Meeting, AIAA Paper 2011-0855, 2011.

-[9] Bermejo-Moreno, I., Campo, L., Larsson, J., Bodart, J., Helmer, D., and Eaton, J. K., "Confinement Effects in Shock Wave/Turbulent Boundary Layer Interactions Through Wall-Modelled Large-Eddy Simulations," Journal of Fluid Mechanics, Vol. 758, Oct. 2014, pp. 5-62.

[10] Ducros, F., Ferrand, V., Nicoud, F., Weber, C., Darracq, D., Gacherieu, C., and Poinsot, T., "Large-Eddy Simulation of Shock-Turbulence Interaction," Journal of Computational Physics, Vol. 152, No. 2, 1999, pp. 517-549.

[11] Reda, D. C., and Murphy, J. D., "Sidewall Boundary-Layer Influence on Shock Wave / Turbulent Boundary-Layer Interactions," AIAA Journal, Vol. 11, No. 10, 1973, pp. 1367-1368.

[12] Bruce, P. J. K., Burton, D. M. F., Titchener, N. A., and Babinsky, H., "Corner Effect and Separation in Transonic Channel Flows," Journal of Fluid Mechanics, Vol. 679, May 2011, pp. 247-262.

[13] Benek, J. A., Suchyta, C. J., and Babinsky, H., "The Effect of Wind Tunnel Size and Shock Strength on Incident Shock Boundary Layer Interaction Experiments," 7th Annual Shock Wave/Boundary Layer Interaction (SWBLI) Flow Control and Modeling Workshop, AIAA Paper 2014-3336, 2014.

[14] Humble, R. A., Scarano, F., and Van Oudheusden, B. W., "Particle Image Velocimetry Measurements of a Shock Wave/Turbulent Boundary Layer Interaction," Experiments in Fluids, Vol. 43, No. 2, 2007, pp. 173-183.

[15] Helmer, D. B., "Measurements of a Three-Dimensional ShockBoundary Layer Interaction," Ph.D. Theses, Stanford Univ., Stanford, CA, 2011.

[16] Helmer, D. B., Campo, L. M., and Eaton, J. K., "Three-Dimensional Features of a Mach 2.1 Shock/Boundary Layer Interaction," Experiments in Fluids, Vol. 53, Aug. 2012, pp. 1347-1368.

[17] Adamson, T. C., and Messiter, A. F., "Analysis of Two-Dimensional Interactions Between Shock Waves And Boundary Layers," Annual Review of Fluid Mechanics, Vol. 12, Jan. 1980, pp. 103-138.

[18] Zheltovodov, A. A., "Shock Waves/Turbulent Boundary-Layer Interactions-Fundamental Studies and Applications," 27th AIAA Fluid Dynamics Conference, AIAA Paper 1996-1977, 1996.

[19] Beresh, S. J., Clemens, N. T., Dolling, D. S., and Comninos, M., "Investigation of the Causes of Large-Scale Unsteadiness of ShockInduced Separated Flow Using Laser Imaging," 35th Aerospace Sciences Meeting, AIAA Paper 1997-0064, 1997.

[20] Kubota, H., and Stollery, J. L., "An Experimental Study of the Interaction Between a Glancing Shock Wave and a Turbulent Boundary Layer," Journal of Fluid Mechanics, Vol. 116, March 1982, pp. 431-458.

- [21] Délery, J., and Dussauge, J.-P., "Some Physical Aspects of Shock Wave/ Boundary Layer Interactions," Shock Waves, Vol. 19, July 2009, pp. 453-468.

[22] "Shock-Wave/Boundary-Layer-Interactions in Supersonic and Hypersonic Flows," AGARD-R-792, 1993.

[23] Alvi, F. S., and Settles, G. S., "Physical Model of the Swept Shock Wave/Boundary-Layer Interaction Flowfield," AIAA Journal, Vol. 30, No. 9, 1992, pp. 2252-2258.

[24] Lu, F. K., "Quasiconical Free Interaction Between a Swept Shock and a Turbulent Boundary Layer," AIAA Journal, Vol. 31, No. 4, April 1993, pp. 686-692.

[25] Panaras, A. G., "The Effect of the Structure of Swept-Shock-Wave/ Turbulent-Boundary-Layer Interactions on Turbulence Modelling," Journal of Fluid Mechanics, Vol. 338, May 1997, pp. 203-230.

[26] Burton, D. M. F., and Babinsky, H., "Corner Separation Effects for Normal Shock Wave/Turbulent Boundary Layer Interactions in Rectangular Channels," Journal of Fluid Mechanics, Vol. 707, Aug. 2012, pp. 287-306.

[27] Dolling, D. S., "Fifty Years of Shock-Wave/Boundary-Layer Interaction Research: What Next?" AIAA Journal, Vol. 39, No. 8, Aug. 2001, pp. 1517-1531.

[28] Clemens, N. T., and Narayanaswamy, V., "Low-Frequency Unsteadiness of Shock Wave/Turbulent Boundary Layer Interactions," Annual Review of Fluid Mechanics, Vol. 46, Jan. 2014, pp. 469-492.

[29] Eagle, W. E., Driscoll, J. F., and Benek, J. A., "Experimental Investigation of Corner Flows in Rectangular Supersonic Inlets with 3-D Shock-Boundary Layer Effects," 49th AIAA Aerospace Sciences Meeting including the New Horizons Forum and Aerospace Exposition, AIAA Paper 2011-0857, 2011.
[30] Morgan, B., Kawai, S., and Lele, S. K., "A Parametric Investigation of Oblique Shockwave/Turbulent Boundary Layer Interaction Using LES," 41 st AIAA Fluid Dynamics Conference and Exhibit, AIAA Paper 2011-3430, 2011.

[31] Batcho, P., and Sullivan, J., "The 3-D Flowfield in a Supersonic Shock Boundary Layer Corner Interaction," AIAA 26th Aerospace Sciences Meeting, AIAA Paper 1988-0307, 1988.

[32] Cresci, R. J., Rubin, S. G., Nardo, C. T., and Lin, T. C., "Hypersonic Interaction Along a Rectangular Corner," AIAA Journal, Vol. 7, No. 12, 1969, pp. 2241-2247.

[33] Doerffer, P., and Dallmann, U., "Reynolds Number Effect on Separation Structures at Normal Shock Wave/Turbulent Boundary-Layer Interaction," AIAA Journal, Vol. 27, No. 9, 1988, pp. 1206-1213.

[34] Bruce, P. J. K., Babinsky, H., Tartinville, B., and Hirsch, C., "Corner Effect and Asymmetry in Transonic Channel Flows," AIAA Journal, Vol. 49, No. 11, Nov. 2011, pp. 2382-2392.

[35] Handa, T., Masuda, M., and Matsuo, K., "Three-Dimensional Normal Shock-Wave/Boundary-Layer Interaction in a Rectangular Duct," AIAA Journal, Vol. 43, No. 10, 2005, pp. 2182-2187.

[36] Humble, R. A., Elsinga, G. E., Scarano, F., and van Oudheusden, B. W., "Three-Dimensional Instantaneous Structure of a Shock Wave/ Turbulent Boundary Layer Interaction," Journal of Fluid Mechanics, Vol. 622, Feb. 2009, pp. 33-62.

[37] Morgan, B., Duraisamy, K., Nguyen, N., Kawai, S., and Lele, S. K., "Flow Physics and RANS Modelling of Oblique Shock/Turbulent Boundary Layer Interaction," Journal of Fluid Mechanics, Vol. 729, July 2013, pp. 231-284.

[38] Wang, B., Sandham, N. D., Hu, Z., and Liu, W., "Numerical Study of Oblique Shock-Wave/Boundary-Layer Interaction Considering Sidewall Effects," Journal of Fluid Mechanics, Vol. 767, Feb. 2015, pp. 526-561.

[39] Lapsa, A. P., "Experimental Study of Passive Ramps for Control of Shock-Boundary Layer Interactions," Ph.D. Thesis, Univ. of Michigan, Ann Arbor, MI, 2009.

[40] Lapsa, A. P., and Dahm, W. J. A., "Stereo Particle Image Velocimetry of Nonequilibrium Turbulence Relaxation in a Supersonic Boundary Layer," Experiments in Fluids, Vol. 50, June 2010, pp. 89-108.

[41] Adrian, R. J., and Westerweel, J., Particle Image Velocimetry, Cambridge Univ. Press, New York, 2010, pp. 178-195.

[42] Garcia, D., "Robust Smoothing of Gridded Data in One and Higher Dimensions with Missing Values," Computational Statistics and Data Analysis, Vol. 54, April 2010, pp. 1167-1178.

[43] Moisy, F., "PIVMAT Toolbox 3.02," 2013.

[44] Edinburgh Mathematical Society, http://journals.cambridge. org/action/displayAbstract?fromPage $=$ online \&aid $=3140568 \&$ fileld $=$ S001309150000359X.

[45] McKeon, B. J., and Smits, A. J., "Static Pressure Correction in High Reynolds Number Fully Developed Turbulent Pipe Flow," Measurement Science and Technology, Vol. 13, Oct. 2002, pp. 1608-1614.

[46] Chue, S. H., "Pressure Probes for Fluid Measurement," Progress in Aerospace Sciences, Vol. 16, Jan. 1975, pp. 147-223.

[47] Shaw, R., "The Influence of Hole Dimensions on Static Pressure Measurements," Journal of Fluid Mechanics, Vol. 7, 1960, pp. 550-564.

[48] Sebacher, D. I., and Lee, L. P., "NASA Technical Note Crossflow in Two-Dimensional," NASA, TN-D-7999, 1975.

[49] Babinsky, H., and Harvey, J. K., Shock Wave-Boundary-Layer Interactions, Cambridge Univ. Press, New York, 2011, pp. 137-201.

[50] Délery, J. M., and Marvin, J. G., "Shock-Wave Boundary Layer Interactions," AGARD Monograph AG-280, 1986.

[51] Korkegi, R. H., "A Simple Correlation for Incipient Turbulent Boundary-Layer Separation due to a Skewed Shock Wave," AIAA Journal, Vol. 11, No. 11, 1973, pp. 1578-1579.

-[52] Knight, D. D., Horstman, C., Bogdonoff, S., and Shapey, B., "Structure of Supersonic Turbulent Flow Past a Sharp Fin," AIAA Journal, Vol. 25, No. 10, Oct. 1987, pp. 1331-1337.

[53] Lu, F. K., Pierce, A. J., Shih, Y., Liu, C., and Li, Q., "Experimental and Numerical Study of Flow Topology Past Micro Vortex Generators," AIAA 40th Fluid Dynamics Conference and Exhibit, AIAA Paper 20104463, 2010.

[54] Mccormick, D. C., "Shock/Boundary-Layer Interaction Control with Vortex Generators and Passive Cavity," AIAA Journal, Vol. 31, No. 1, Jan. 1993, pp. 91-96.

[55] Panton, R. L., "Incompressible Flow," Wiley, New York, July 2013, Chap. 4.

[56] Chakraborty, P., Balachandar, S., and Adrian, R. J., "On the Relationships Between Local Vortex Identification Schemes," Journal of Fluid Mechanics, Vol. 535, July 2005, pp. 189-214.

[57] Jeong, J., and Hussain, F., "On the Identification of a Vortex," Journal of Fluid Mechanics, Vol. 285, Feb. 1995, pp. 69-94. 
[58] Horiuti, K., and Takagi, Y., "Identification Method for Vortex Sheet Structures in Turbulent Flows," Physics of Fluids, Vol. 17, No. 12, 2005, pp. 1-4.

[59] Graftieaux, L., Michard, M., and Grosjean, N., "Combining PIV, POD and Vortex Identification Algorithms for the Study of Unsteady Turbulent Swirling Flows," Measurement Science and Technology, Vol. 12, Aug. 2001, pp. 1422-1429.

60] Davis, D. O., and Gessner, F. B., "Further Experiments on Supersonic Turbulent Flow Development in a Square Duct," AIAA Journal, Vol. 27, No. 8, 1989, pp. 1023-1030.

[61] Kornilov, V. I., "Correlation of the Separation Region Length in Shock Wave/Channel Boundary Layer Interaction," Experiments in Fluids, Vol. 23, , No. 6, 1997, pp. 489-497.

[62] Gessner, F. B., Eppich, H. M., and Lund, E. G., "Near-wall turbulent flows : proceedings of the International Conference on Near-Wall Turbulent Flows," Near-Wall Turbulent Flows, Elsevier Science, Philadelphia, PA, 1993, pp. 965-975.

[63] Wang, K. C., "Separation Patterns of Boundary Layer over an Inclined Body of Revolution," AIAA Journal, Vol. 10, No. 8, Aug. 1972, pp. 1044-1050.
[64] Wang, K. C., and Diego, S., "On the Disputes About Open Separation," AIAA 21 st Aerospace Sciences Meeting, AIAA Paper 1983-0296, 1983.

[65] Tobak, M., and Peake, D. J., "Topology of Three-Dimensional Separated Flows," Annual Review of Fluid Mechanics, Vol. 14, Jan. 1982, pp. 61-85.

[66] Wu, J. Z., Tramel, R. W., Zhu, F. L., and Yin, X. Y., "A Vorticity Dynamics Theory of Three-Dimensional Flow Separation," Physics of Fluids, Vol. 12, No. 8, 2000, pp. 1932-1954.

[67] Tropea, C., and Martinuzzi, R., "The Flow Around Surface-Mounted, Prismatic Obstacles Placed in a Fully Developed Channel Flow," Journal of Fluids Engineering, Vol. 115, No. 1, 1993, pp. 85-92.

[68] Chou, J. H., and Chao, S. Y., "Branching of a Horseshoe Vortex Around Surface-Mounted Rectangular Cylinders," Experiments in Fluids, Vol. 28, May 2000, pp. 394-402.

F. Alvi Associate Editor 